\title{
Random Matrices and Subexponential Operator Spaces
}

\author{
by \\ Gilles Pisier \\ Texas A\&M University \\ College Station, TX 77843, U. S. A. \\ and \\ Université Paris VI \\ IMJ, Equipe d'Analyse Fonctionnelle, Case 186, \\ 75252 Paris Cedex 05, France
}

August 24, 2018

\begin{abstract}
We introduce and study a generalization of the notion of exact operator space that we call subexponential. Using Random Matrices we show that the factorization results of Grothendieck type that are known in the exact case all extend to the subexponential case, but we exhibit (a continuum of distinct) examples of non-exact subexponential operator spaces, as well as a $C^{*}$-algebra that is subexponential with constant 1 but not exact. We also show that $O H, R+C$ and $\max \left(\ell_{2}\right)$ (or any other maximal operator space) are not subexponential.
\end{abstract}

In [12, 26] operator space versions of Grothendieck's theorem were proved in the form of a special factorization property for (jointly) completely bounded bilinear forms on $E \times F$ when $A, B$ are $C^{*}$ algebras and $E \subset A, F \subset B$ are exact operator subspaces. In particular, when $E=A, F=B$ this was proved for exact $C^{*}$-algebras. In [11] this last result was extended to arbitrary $C^{*}$-algebras. A remarkable, considerably simpler proof was recently given in [27]. In the case of "exact" subspaces $E \subset A, F \subset B$, this recent proof from [27] deduces the result of [26] directly from that of [12]. In this paper we introduce a larger class of operator spaces, that we call "subexponential", for which the same Grothendieck type factorization property from [12, 26] still holds. The known examples of non-exact operator spaces turn out to be also non-subexponential, but in $\$ 8$ an example is constructed showing that the new class is strictly larger than that of exact operator spaces.

The definition of "subexponential" involves the growth of a sequence of integers $N \mapsto K_{E}(N, C)$ attached to an operator space $E$ (and a constant $C>1$ ), in a way that is similar but seems different from the number $k_{E}(N, C)$ introduced by us in [25]. We denote by $K_{E}(N, C)$ the smallest $K$ such that there is a linear embedding $f: E \rightarrow M_{K}$ satisfying

$$
\forall x \in M_{N}(E) \quad\|(I d \otimes f)(x)\|_{M_{N}\left(M_{K}\right)} \leq\|x\|_{M_{N}(E)} \leq C\|(I d \otimes f)(x)\|_{M_{N}\left(M_{N}\right)} .
$$

The latter sequence is bounded iff $E$ is $C$-exact while it is such that $\log K_{E}(N, C) / N \rightarrow 0$ iff $E$ is $C$ subexponential. For the non-exact $C^{*}$-algebra $A$ constructed in $\$ 7$ we have polynomial growth: we have $K_{E}(N, 1+\varepsilon) \in O\left(N^{d}\right)$ for any finite dimensional $E \subset A$ for some $d$ depending on $E$ and $\varepsilon>0$. For the non-exact example in $₫ 8$, we have $K_{E}(N, 2+\varepsilon) \in O\left(N^{2}\right)$ and also $K_{E}(N, 2+\varepsilon) \geq c \sqrt{N}$. There is a notion of "subexponential constant" analogous to the exactness constant, and we give estimates from below (of the same order) of that constant for the same examples $\left(O H_{n}, R_{n}+C_{n}\right.$ or maximal spaces) for which lower bounds of the exactness constant are known. 
To tackle subexponentiality, we make crucial use of Gaussian random matrices and particularly of Haagerup and Thorbjørnsen's [8]. Let $Y^{(N)}$ denote a random $N \times N$-matrix with i.i.d. complex Gaussian entries $Y^{(N)}(i, j)$ with $\mathbb{E} Y^{(N)}(i, j)=0$ and $\mathbb{E}\left|Y^{(N)}(i, j)\right|^{2}=1 / N$, and let $\left(Y_{j}^{(N)}\right)$ be a sequence of i.i.d. copies of $Y^{(N)}$ on some probability space $(\Omega, \mathbb{P})$. Let $E \subset B(H)$ be an operator space. For any $\left(a_{1}, \cdots, a_{n}\right) \in E^{n}$ we define

$$
\left\|\left(a_{1}, \cdots, a_{n}\right)\right\|\left\|=\lim \sup _{N \rightarrow \infty}\right\| \sum_{1}^{n} Y_{j}^{(N)} \otimes a_{j} \|,
$$

where-here and below-the norm is the minimal (or spatial) tensor norm (here this is simply the norm of $M_{N}(B(H))$. Note that this is non-random. Indeed, by concentration of measure (see \$2) we have almost surely

$$
\lim \sup _{N \rightarrow \infty}\left|\left\|\sum_{1}^{n} Y_{j}^{(N)} \otimes a_{j}\right\|-\mathbb{E}\left\|\sum_{1}^{n} Y_{j}^{(N)} \otimes a_{j}\right\|\right|=0,
$$

and hence

$$
\left\|\left(a_{1}, \cdots, a_{n}\right)\right\| \mid=\lim \sup _{N \rightarrow \infty} \mathbb{E}\left\|\sum_{1}^{n} Y_{j}^{(N)} \otimes a_{j}\right\| .
$$

The main result of Haagerup and Thorbjørnsen's [9] implies that if $E$ is exact with constant 1 then

$$
\left\|\left(a_{1}, \cdots, a_{n}\right) \mid\right\|=\left\|\sum c_{j} \otimes a_{j}\right\|
$$

where $\left(c_{j}\right)$ is a free circular sequence in Voiculescu's sense, and the limsup is actually almost surely a limit. Indeed, it can be shown rather easily that

$$
\left\|\sum c_{j} \otimes a_{j}\right\| \leq \liminf _{N \rightarrow \infty}\left\|\sum_{1}^{n} Y_{j}^{(N)} \otimes a_{j}\right\|
$$

holds almost surely for any E. By (0.2) this boils down to

$$
\left\|\sum c_{j} \otimes a_{j}\right\| \leq \liminf _{N \rightarrow \infty} \mathbb{E}\left\|\sum_{1}^{n} Y_{j}^{(N)} \otimes a_{j}\right\| .
$$

These lower bounds are easy to deduce from the well known weak convergence of $\left(Y_{j}^{(N)}\right)$ to a free circular system $\left(c_{j}\right)$ due to Voiculescu (see [29]), originating in Wigner's famous result for a single matrix $\left(Y^{(N)}\right)$. This weak convergence asserts that if $\tau_{N}$ (resp. $\tau$ ) denotes the normalized trace on $M_{N}$ (resp. on the von Neumann algebra generated by $\left(c_{j}\right)$ ), we have a.s.

$$
\lim _{N \rightarrow \infty} \tau_{N}\left(P\left(Y_{j}^{(N)}\right)\right)=\tau\left(P\left(c_{j}\right)\right)
$$

for any polynomial $P$ in the non-commuting variables $c_{j}, c_{j}^{*}$. The term $*$-polynomial would be probably less abusive: Here $P\left(Y_{j}^{(N)}\right) \in M_{N}$ denotes the matrix obtained after substituting $\left(Y_{j}^{(N)}, Y_{j}^{(N)^{*}}\right)$ to $\left(c_{j}, c_{j}^{*}\right)$ in $P\left(c_{j}\right)$. Equivalently, for almost all $\omega$ in our probability space $\Omega$, all the $\tau_{N}$-moments combining the matrices $\left(Y_{j}^{(N)}(\omega)\right)$ and their adjoints converge to the analogous $\tau$-moments in $\left(c_{j}\right)$ and their adjoints. 
The above (0.4) from 9] was recently extended to unitary matrices (and a few other cases) by Collins and Male, see [3]. In that case, $Y^{(N)}$ is uniformly distributed over the unitary group $U(N)$ of all $N \times N$ unitary matrices, and $\left(c_{j}\right)$ has to be replaced by a free family of Haar unitaries.

If $E$ is $C$-exact, then [9] implies $\left\|\left(a_{1}, \cdots, a_{n}\right)\right\| \leq C C \sum c_{j} \otimes a_{j} \|$. A fortiori this implies the following result proved in [8] (prior to [9]): If $E$ is $C$-exact, then

$$
\forall n \forall\left(a_{1}, \cdots, a_{n}\right) \in E^{n} \quad\left\|\left(a_{1}, \cdots, a_{n}\right)\right\| \| \leq 2 C \max \left\{\left\|\left(\sum a_{j}^{*} a_{j}\right)^{1 / 2}\right\|,\left\|\left(\sum a_{j} a_{j}^{*}\right)^{1 / 2}\right\|\right\} .
$$

The starting point of our study of subexponential spaces is the observation (that we made several years ago after reading [8]) that (0.8) remains valid if $E$ is $C$-subexponential. More precisely, we insist on formulating universal bounds that correspond to an estimate of the speed of convergence in (0.4) or (0.8). Such inequalities, that are crucial in the sequel, are implicit in [8, 9, 10]. For instance, given $\varepsilon>0$ there is $\gamma_{\varepsilon}>0$ such that:

For any $E$ and any $a_{1}, \cdots, a_{n} \in E$, any $\varepsilon>0$ we have

$\mathbb{E}\left\|\sum_{1}^{n} Y_{j}^{(N)} \otimes a_{j}\right\| \leq C(1+\varepsilon)\left(2+\gamma_{\varepsilon}\left(\frac{\log \left(K_{E}(N, C)\right)+1}{N}\right)^{1 / 2}\right) \max \left\{\left\|\left(\sum a_{j}^{*} a_{j}\right)^{1 / 2}\right\|,\left\|\left(\sum a_{j} a_{j}^{*}\right)^{1 / 2}\right\|\right\}$.

This can be deduced from [8]. We include a quick proof based as [8] on the Wick formula but taking advantage of a result of Buchholz [2].

For our application to Grothendieck's inequality (0.8) is enough. This motivates the definitions of "tight" and "completely tight" in Definition 3.1 below. However, for other $C^{*}$-algebraic questions, one needs the more refined (0.4) from [9]. One can also deduce from [9] an estimate of the speed of convergence, but as the right hand side is more precise the error term is less well controled. Thus one obtains a bound of the form

$$
\mathbb{E}\left\|\sum_{1}^{n} Y_{j}^{(N)} \otimes a_{j}\right\| \leq C(1+\varepsilon)\left(1+\gamma_{\varepsilon}^{\prime} \frac{K_{E}(N, C)^{4}}{N}\right)\left\|\sum c_{j} \otimes a_{j}\right\|,
$$

where $\gamma_{\varepsilon}^{\prime}$ may now depend on both $\varepsilon$ and $n$.

More generally (see (7.13) below), if we replace $\sum_{1}^{n} Y_{j}^{(N)} \otimes a_{j}$ and $\sum_{1}^{n} c_{j} \otimes a_{j}$ by polynomials $P\left(Y_{j}^{(N)}\right.$ ) and $P\left(c_{j}\right)$ of degree $d$ in the non-commuting variables $c_{j}, c_{j}^{*}$, then the analogous inequality can be deduced from [10], but now $\gamma_{\varepsilon}^{\prime}$ is allowed to depend on $d$ in addition to $\varepsilon$ and $n$.

Using the concentration of measure method, one can deduce from these inequalities surprisingly strong almost sure consequences, via the following known Lemma (see 92 for the proof).

Lemma 0.1. Consider the following event: let $\Omega_{\varepsilon, n}(k, N) \subset \Omega$ denote the set of $\omega \in \Omega$ such that

$$
\forall a_{j} \in M_{k} \quad\left|\left\|\sum_{1}^{n} Y_{j}^{(N)}(\omega) \otimes a_{j}\right\|-\mathbb{E}\left\|\sum_{1}^{n} Y_{j}^{(N)} \otimes a_{j}\right\|\right| \leq \varepsilon \mathbb{E}\left\|\sum_{1}^{n} Y_{j}^{(N)} \otimes a_{j}\right\| .
$$

Then for any $\varepsilon>0$ there is a constant $c_{\varepsilon}>0$ such that whenever $N \geq c_{\varepsilon} n k^{2}$ we have

$$
\mathbb{P}\left(\Omega_{\varepsilon, n}(k, N)\right) \geq 1-\exp -c^{\prime} \varepsilon^{2} N
$$

where $c^{\prime}>0$ is an absolute numerical constant.

Since $\Omega_{\varepsilon, n}\left(k^{\prime}, N\right) \subset \Omega_{\varepsilon, n}(k, N)$ for any $k \leq k^{\prime}$ we may focus on the largest $k$ such that $N \geq c_{\varepsilon} n k^{2}$, i.e. on $k_{\varepsilon, n}(N)=\left[\left(c_{\varepsilon}^{-1} n^{-1} N\right)^{1 / 2}\right]$ (the main point is $k_{\varepsilon, n}(N) \approx N^{1 / 2}$ ), and rewrite (0.11) as

$$
\mathbb{P}\left(\Omega_{\varepsilon, n}\left(k_{\varepsilon, n}(N), N\right)^{c}\right) \leq \exp -c^{\prime} \varepsilon^{2} N
$$


Since $\sum_{N} \exp -c^{\prime} \varepsilon^{2} N<\infty$, we find that, for any $n$ and $\varepsilon>0$, we have

$$
\mathbb{P}\left(\liminf _{N \rightarrow \infty} \Omega_{\varepsilon, n}\left(k_{\varepsilon, n}(N), N\right)\right)=1 .
$$

In otherwords, for almost all $\omega$, the property in (0.10) with $k=k_{\varepsilon, n}(N)$ holds for all $N$ large enough.

This explains why control of the moment as in (0.9) leads to rather strong almost sure consequences. For instance (0.9) implies that if $K_{E}(N, 1+\varepsilon) \in o\left(N^{1 / 4}\right)$ for any $\varepsilon>0$, then for almost all $\omega$

$$
\lim _{N \rightarrow \infty}\left\|\sum_{1}^{n} Y_{j}^{(N)}(\omega) \otimes a_{j}\right\|=\left\|\sum c_{j} \otimes a_{j}\right\|
$$

More generally, consider a sequence of integers $K(N)$ such that $K(N) \in o\left(N^{1 / 4}\right)$, and assume that we have $n$-tuples $a^{(N)}=\left(a_{j}^{(N)}\right) \in M_{K(N)}^{n}$ such that

$$
\lim _{N \rightarrow \infty}\left\|\sum_{1}^{n} c_{j} \otimes a_{j}^{(N)}\right\|=\left\|\sum c_{j} \otimes a_{j}\right\| .
$$

Then Lemma 0.1 implies that for almost all $\omega$

$$
\lim _{N \rightarrow \infty}\left\|\sum_{1}^{n} Y_{j}^{(N)}(\omega) \otimes a_{j}^{(N)}\right\|=\left\|\sum c_{j} \otimes a_{j}\right\| .
$$

We use this phenomenon in the construction of our non-exact subexponential examples. The $C^{*}$ algebra in $₫ 7$ is the simplest to describe: we just consider for $j \in \mathbb{N}$ the block diagonal sum

$$
u_{j}(\omega)=\oplus_{N \geq 1} Y_{j}^{(N)}(\omega) \in \oplus_{N \geq 1} M_{N}
$$

and we define $A(\omega)$ as the unital $C^{*}$-algebra generated by $\left\{u_{j}(\omega) \mid j \in \mathbb{N}\right\}$ in $\oplus_{N \geq 1} M_{N}$.

Then, for almost all $\omega, A(\omega)$ is subexponential with constant 1 but is not exact.

It seems natural to wonder what becomes of (0.8) or (0.1) when $E$ is no longer assumed exact or subexponential. We propose some leads in this direction in $\$ 9$ below.

This paper is closely linked to [25] where the "growth" of an operator space $E$ is studied via a different number denoted by $k_{E}(N, C)$. There is an obvious upper bound (for a fixed constant $C$ ) $K_{E}(N, C) \leq N k_{E}(N, C)$, so the growth of $K_{E}$ is dominated by that of $k_{E}$, but we know nothing in the converse direction. Various other questions are mentioned at the end of $\$$.

\section{Background on Operator Spaces}

By definition, an operator space is just a closed subspace $E \subset B(H)$ of the space of bounded operators on a Hilbert space $H$. For any $N \geq 1$, we denote by $M_{N}(E)$ the space of $N \times N$ matrices with entries in $E$.

In operator space theory, the space $E$ is equipped not only with the induced norm, but also with the sequence of norms induced on $M_{N}(E)$ by $M_{N}(B(H))$. The space $M_{N}(B(H))$ is here equipped with the norm associated to the identification $M_{N}(B(H)) \simeq B(H \oplus \cdots \oplus H)$.

In this theory, the space $B(E, F)$ of all bounded linear maps $u: E \rightarrow F$ between two operator spaces $E, F$ is replaced by the space $C B(E, F)$ of all the completely bounded (in short c.b.) ones, defined as follows.

For any given $N \geq 1$ we denote by

$$
u_{N}: M_{N}(E) \rightarrow M_{N}(F)
$$


the mapping taking $\left[a_{i j}\right]$ to $\left[u\left(a_{i j}\right)\right]$. Equivalently, using the isomorphisms $M_{N}(E) \simeq M_{N} \otimes E$, $M_{N}(F) \simeq M_{N} \otimes F$ we will identify $u_{N}$ to $I d \otimes u: M_{N} \otimes E \rightarrow M_{N} \otimes E$. The mapping $u$ is called completely bounded (in short c.b.) if $\sup _{N \geq 1}\left\|u_{N}\right\|<\infty$. We then define

$$
\|u\|_{c b}=\sup _{N \geq 1}\left\|u_{N}\right\| .
$$

We say that $E, F$ are completely isomorphic if there is a c.b. isomorphism $u: E \rightarrow F$ with c.b. inverse.

Moreover, if $E, F$ are two operator spaces that are isomorphic as Banach spaces, we set

$$
d_{N}(E, F)=\inf \left\{\left\|u_{N}\right\|\left\|\left(u^{-1}\right)_{N}\right\|\right\}
$$

where the inf runs over all the isomorphisms $u: E \rightarrow F$. We set $d_{N}(E, F)=\infty$ if $E, F$ are not isomorphic. When $N=1$ we recover the usual Banach-Mazur distance.

Similarly, if $E, F$ are completely isomorphic, we set

$$
d_{c b}(E, F)=\inf \left\{\|u\|_{c b}\left\|u^{-1}\right\|_{c b}\right\}
$$

where the inf runs over all the complete isomorphisms $u: E \rightarrow F$.

It is an easy exercise to check that if $E, F$ are of the same finite dimension, we have

$$
d_{c b}(E, F)=\sup _{N \geq 1} d_{N}(E, F) .
$$

Moreover, a simple compactness argument shows that (again if $\operatorname{dim}(E)=\operatorname{dim}(F)<\infty$ ) there is an isomorphism $u: E \rightarrow F$ such that $\|u\|_{c b}\left\|u^{-1}\right\|_{c b}=d_{c b}(E, F)$, and after scaling we may assume e.g. $\|u\|_{c b}=d_{c b}(E, F)$ and $\left\|u^{-1}\right\|_{c b}=1$.

Given a bilinear form $\varphi: E \times F \rightarrow \mathbb{C}$ we define

$$
\varphi_{N}: M_{N}(E) \times M_{N}(F) \rightarrow M_{N} \otimes M_{N} \simeq M_{N}\left(M_{N}\right)
$$

as the bilinear map defined by $\varphi_{N}(y \otimes a, z \otimes b)=y \otimes z \varphi(a, b)$. The form $\varphi$ is called (jointy) c.b. if

$$
\|\varphi\|_{c b}=\sup _{N}\left\|\varphi_{N}\right\|<\infty .
$$

The operator space dual $F^{*}$ of an operator space $F$ is characterized by the fact that for any $E$ and any bilinear form $\varphi: E \times F \rightarrow \mathbb{C}$ the associated linear map $u_{\varphi}: E \rightarrow F^{*}$ satisfies

$$
\left\|u_{\varphi}\right\|_{c b}=\|\varphi\|_{c b}
$$

The existence (for some $\mathcal{H}$ ) of an isometric embedding $F^{*} \subset B(\mathcal{H})$ of the Banach dual $F^{*}$ for which this holds is a consequence of Ruan's fundamental theorem (see [4, 21]).

The following Lemma due to Roger Smith will be very useful.

Lemma 1.1. Let $E \subset M_{K}$ be any operator space. Then for any operator space $X$ and any bounded linear map $u: X \rightarrow E$ we have

$$
\|u\|_{c b}=\left\|u_{K}\right\|
$$

We refer the reader to [4, 21] for a proof of this and for more information on operator spaces.

The row and column spaces $R=\operatorname{span}\left[e_{1 j}\right] \subset B\left(\ell_{2}\right)$ and $C=\operatorname{span}\left[e_{i 1}\right] \subset B\left(\ell_{2}\right)$ are fundamental examples of operator spaces, as well as the finite dimensional versions:

$$
R_{n}=\operatorname{span}\left[e_{1 j}, 1 \leq j \leq n\right] \subset M_{n} \quad C_{n}=\operatorname{span}\left[e_{i 1}, 1 \leq i \leq n\right] \subset M_{n} .
$$


For $a=\left(a_{1}, \cdots, a_{n}\right)$ with $a_{j} \in B(H)$ we denote

$$
\begin{gathered}
\|a\|_{R C}=\max \left\{\left\|\left(\sum a_{j}^{*} a_{j}\right)^{1 / 2}\right\|,\left\|\left(\sum a_{j} a_{j}^{*}\right)^{1 / 2}\right\|\right\}, \\
\|a\|_{R}=\left\|\left(\sum a_{j} a_{j}^{*}\right)^{1 / 2}\right\|, \text { and }\|a\|_{C}=\left\|\left(\sum a_{j}^{*} a_{j}\right)^{1 / 2}\right\|,
\end{gathered}
$$

so that

$$
\|a\|_{R C}=\max \left\{\|a\|_{R},\|a\|_{C}\right\} .
$$

The reader should observe that when $\operatorname{dim}(H)=N$ then $\|a\|_{R},\|a\|_{C}$ are just short cut notation for the norms in $M_{N}(E)$ respectively for $E=R_{n}, C_{n}$. Indeed, we have $\|a\|_{R}=\left\|\sum a_{j} \otimes e_{1 j}\right\|_{M_{N}\left(R_{n}\right)}$ and $\|a\|_{C}=\left\|\sum a_{j} \otimes e_{j 1}\right\|_{M_{N}\left(C_{n}\right)}$. The norm $\|a\|_{R C}$ corresponds similarly to the span of $\left[e_{1 j} \oplus e_{j 1}, 1 \leq\right.$ $j \leq n]$ in $R_{n} \oplus C_{n}$.

Let $\left(c_{j}\right)$ be a free circular system in a von Neumann algebra $M$ equipped with a normalized trace $\tau$. For any $a_{j} \in M_{k}$ we have

$$
\max \left\{\left\|\left(\sum a_{j}^{*} a_{j}\right)^{1 / 2}\right\|,\left\|\left(\sum a_{j} a_{j}^{*}\right)^{1 / 2}\right\|\right\} \leq\left\|\sum c_{j} \otimes a_{j}\right\| \leq 2 \max \left\{\left\|\left(\sum a_{j}^{*} a_{j}\right)^{1 / 2}\right\|,\left\|\left(\sum a_{j} a_{j}^{*}\right)^{1 / 2}\right\|\right\} .
$$

Indeed, setting $S=\sum c_{j} \otimes a_{j}$ the lower bound follows from the identities $\sum a_{j}^{*} a_{j} \otimes 1=(I d \otimes \tau)\left(S^{*} S\right)$ and $\sum a_{j} a_{j}^{*} \otimes 1=(I d \otimes \tau)\left(S S^{*}\right)$. The upper bound follows from the decomposition of $c_{j}$ as a sum of free creation and annihilation operators. See e.g. [26] for details.

\section{Concentration of measure and Random Matrices}

We will use the term "complex valued Gaussian" random variable for any random variable of the form $g=g^{\prime}+i g^{\prime \prime}$ with $\left(g^{\prime}, g^{\prime \prime}\right)$ independent real valued Gaussian variable such that $\mathbb{E} g^{\prime}=\mathbb{E} g^{\prime \prime}=0$ and $\mathbb{E}\left|g^{\prime}\right|^{2}=\mathbb{E}\left|g^{\prime \prime}\right|^{2}$. Actually all our Gaussian variables will be assumed to have zero mean.

We will denote by $Y^{(N)}$ a random $N \times N$-matrix with i.i.d. complex Gaussian entries with $L_{2}$-norm equal to $N^{-1 / 2}$ and we denote by $\left(Y_{j}^{(N)}\right)$ a sequence of i.i.d. copies of $Y^{(N)}$.

Given an operator space $E \subset B(H)$ and $a=\left(a_{1}, \cdots, a_{n}\right) \in E^{n}$, we will study the $M_{N}(E)$-valued random variable $S_{a}$ defined by

$$
S_{a}=\sum_{1}^{n} Y_{j}^{(N)} \otimes a_{j} .
$$

This is a Gaussian variable with values in a Banach space, to which the known concentration of measure inequalities, that we now recall, can be applied.

Let $f: \mathbb{R}^{d} \rightarrow \mathbb{R}$ be a function such that

$$
\sigma=\sup \left\{\frac{|f(x)-f(y)|}{\|x-y\|_{2}} \mid x \neq y \in \mathbb{R}^{d}\right\}<\infty
$$

where $\|\cdot\|_{2}$ denotes the Euclidean norm on $\mathbb{R}^{d}$. Then, if $\mathbb{P}$ is the canonical Gaussian measure on $\mathbb{R}^{d}$, we have

$$
\forall t>0 \quad \mathbb{P}\{|f-\mathbb{E} f|>t\} \leq 2 \exp -t^{2} / 2 \sigma^{2} .
$$

See [13] for details. Note that it is much easier (see [18]) to prove this with an upper bound of the form $2 \exp -c t^{2} / \sigma^{2}$ for some absolute numerical constant $c$, and, as often, this is enough for our 
purposes.

In particular, we may view $Y^{(N)}$ as an $M_{N}$-valued variable defined on $\mathbb{R}^{2 N^{2}}$ of the form

$$
Y^{(N)}=\sum_{i j}(2 N)^{-1 / 2}\left(g_{i j}^{\prime}+\sqrt{-1} g_{i j}^{\prime \prime}\right) e_{i j}
$$

Applying the above to $f=\left\|Y^{(N)}\right\|$ on $\mathbb{R}^{2 N^{2}}$ we find $\sigma=(2 N)^{-1 / 2}$ and

$$
\forall t>0 \quad \mathbb{P}\left\{\left|\left\|Y^{(N)}\right\|-\mathbb{E}\left\|Y^{(N)}\right\|\right|>t\right\} \leq 2 \exp -N t^{2} .
$$

More generally, if we take $f=\left\|S_{a}\right\|$ (here the norm is in $M_{N}(E)$ ) on $\mathbb{R}^{2 n N^{2}}$ we find

$$
\sigma=(2 N)^{-1 / 2} \sup \left\{\left.\left\|\sum z_{j} a_{j}\right\|\left|z_{j} \in \mathbb{C}, \sum\right| z_{j}\right|^{2} \leq 1\right\} .
$$

Note that with the above notation (1.1) we have

$$
\sup \left\{\left.\left\|\sum z_{j} a_{j}\right\|\left|z_{j} \in \mathbb{C}, \sum\right| z_{j}\right|^{2} \leq 1\right\} \leq \min \left\{\|a\|_{R},\|a\|_{C}\right\} \leq \max \left\{\|a\|_{R},\|a\|_{C}\right\}=\|a\|_{R C}
$$

and hence if we assume $\|a\|_{R C} \leq 1$ we find again

$$
\forall t>0 \quad \mathbb{P}\left\{\left|\left\|S_{a}\right\|-\mathbb{E}\left\|S_{a}\right\|\right|>t\right\} \leq 2 \exp -N t^{2} .
$$

In particular, by the classical Borel-Cantelli argument, this implies that almost surely

$$
\lim \sup _{N \rightarrow \infty}\left|\left\|S_{a}\right\|-\mathbb{E}\left\|S_{a}\right\|\right|=0 .
$$

Proof of Lemma 0.1. Again let $f=\left\|S_{a}\right\|$. We first claim that

$$
(2 N)^{1 / 2} \sigma \mathbb{E}\left\|Y^{(N)}\right\| \leq \mathbb{E}\left\|S_{a}\right\| .
$$

Note that for any linear form $\xi$ such that $\|\xi\|_{E^{*}} \leq 1$, we have $(I d \otimes \xi)\left(S_{a}\right)=\sum_{1}^{n} Y_{j}^{(N)} \xi\left(a_{j}\right)$, and hence $\mathbb{E}\left\|(I d \otimes \xi)\left(S_{a}\right)\right\|=\left(\sum_{1}^{n}\left|\xi\left(a_{j}\right)\right|^{2}\right)^{1 / 2} \mathbb{E}\left\|Y^{(N)}\right\|$. Note that for this particular choice of $f$ we have $\sup _{\xi \in E^{*}}\left(\sum_{1}^{n}\left|\xi\left(a_{j}\right)\right|^{2}\right)^{1 / 2}=\sup \left\{\left.\left\|\sum z_{j} a_{j}\right\|\left|z_{j} \in \mathbb{C}, \sum\right| z_{j}\right|^{2} \leq 1\right\}$. Thus taking the supremum of $\mathbb{E}\left\|(I d \otimes \xi)\left(S_{a}\right)\right\|$ over all $\|\xi\|_{E^{*}} \leq 1$ we find our claim. Note that $\left\|Y^{(N)}\right\| \geq\left(\sum_{1}^{N}\left|Y_{1 j}^{(N)}\right|^{2}\right)^{1 / 2}$ and hence $\mathbb{E}\left\|Y^{(N)}\right\| \geq \gamma(1)$ where $\gamma(1)=\|g\|_{1}$ for any complex Gaussian variable $g$ normalized in $L_{2}$. Thus we obtain

$$
(2 N)^{1 / 2} \gamma(1) \sigma \leq \mathbb{E}\left\|S_{a}\right\| .
$$

Let $\mathcal{N}$ be a $\delta$-net in the unit ball of $M_{k}^{n}$ equipped with the norm $a \mapsto \mathbb{E}\left\|S_{a}\right\|$. Since $\operatorname{dim}_{\mathbb{R}}\left(M_{k}^{n}\right)=$ $2 n k^{2}$, we know (cf. e.g. [5, p. 58] or [19, p. 49]) that there is such a net with $|\mathcal{N}| \leq(1+2 / \delta)^{2 n k^{2}}$. By (2.1) for each $a \in \mathcal{N}$ we have

$$
\mathbb{P}\left\{\left|\left\|S_{a}\right\|-\mathbb{E}\left\|S_{a}\right\|\right|>\varepsilon \mathbb{E}\left\|S_{a}\right\|\right\} \leq 2 \exp -\varepsilon^{2} N \gamma(1)^{2}
$$

and hence if we set

$$
\Omega^{\prime}=\left\{\exists a \in \mathcal{N}\left|\left\|S_{a}\right\|-\mathbb{E}\left\|S_{a}\right\|\right|>\varepsilon \mathbb{E}\left\|S_{a}\right\|\right\}
$$

we have

$$
\mathbb{P}\left(\Omega^{\prime}\right) \leq 2(1+2 / \delta)^{2 n k^{2}} \exp -\varepsilon^{2} N \gamma(1)^{2} \leq \exp \left(4 n k^{2} / \delta-\varepsilon^{2} N \gamma(1)^{2}\right) .
$$

To simplify, let us take $\delta=\varepsilon<1$ and assume that $\varepsilon^{2} N \gamma(1)^{2} \geq 8 n k^{2} / \delta$, which boils down to $8 \varepsilon^{3} n k^{2} \leq N$. We have then

$$
\mathbb{P}\left(\Omega^{\prime}\right) \leq 2 \exp -\varepsilon^{2} N \gamma(1)^{2} / 2
$$

and for any $\omega \in \Omega^{\prime}$

$$
\forall a \in \mathcal{N} \quad(1-\varepsilon) \mathbb{E}\left\|S_{a}\right\| \leq\left\|S_{a}(\omega)\right\| \leq(1+\varepsilon) \mathbb{E}\left\|S_{a}\right\|
$$

but by a well known argument (see e.g.[19, p.49]) this implies for the same $\omega$

$$
\forall a \in M_{k}^{n} \quad(1-3 \varepsilon)(1-\varepsilon)^{-1} \mathbb{E}\left\|S_{a}\right\| \leq\left\|S_{a}(\omega)\right\| \leq(1+\varepsilon)(1-\varepsilon)^{-1} \mathbb{E}\left\|S_{a}\right\|
$$

so the conclusion follows by a straightforward adjustment of $c_{\varepsilon}$ and $c_{\varepsilon}^{\prime}$. 


\section{Operator space versions of Grothendieck's theorem}

Our goal is to study a generalization of the notion of exact operator space for which the version of Grothendieck's factorization theorem obtained in [26] is still valid. The latter asserts that, if $E, F$ are exact operator spaces (assumed separable for simplicity), any c.b. map from $E$ to $F^{*}$ factors through $R \oplus C$. The later proofs of [11] and [27] deduce the full force of the factorization from an apparently weaker inequality. This motivates the following

Definition 3.1. Let $C \geq 1$ be a constant. We will say that an operator space $E$ is $C$-tight if for any $n$ and any $a_{1}, \cdots, a_{n} \in E$ we have

$$
\limsup _{N \rightarrow \infty} \mathbb{E}\left\|\sum_{1}^{n} Y_{j}^{(N)} \otimes a_{j}\right\| \leq C\|a\|_{R C} .
$$

We will say that $E$ is completely $C$-tight if all the spaces $M_{N}(E)(N \geq 1)$ are $C$-tight. We will say that $E$ is tight (resp. completely tight) if it is $C$-tight (resp. completely $C$-tight) for some $C$.

With this terminology, we can refomulate the starting point of [12] like this:

Lemma 3.2. If two operator spaces $E, F$ are respectively $C_{E}$-tight and $C_{F}$-tight (for some constants $\left.C_{E}, C_{F}\right)$ then any $u \in C B\left(E, F^{*}\right)$, with associated bilinear form $\varphi$, satisfies for any $a_{1}, \cdots, a_{n} \in E$ and $b_{1}, \cdots, b_{n} \in F$

$$
\left|\sum \varphi\left(a_{j}, b_{j}\right)\right|=\left|\sum\left\langle u\left(a_{j}\right), b_{j}\right\rangle\right| \leq \lambda\|a\|_{R C}\|b\|_{R C}
$$

where $\lambda=C_{E} C_{F}\|u\|_{c b}$.

Proof. Let $S_{a}^{(N)}=\sum_{1}^{n} Y_{j}^{(N)} \otimes a_{j}$ and $T_{b}^{(N)}=\sum_{1}^{n} \overline{Y_{j}^{(N)}} \otimes b_{j}$. Let $\varphi: E \times F \rightarrow \mathbb{C}$ be the c.b. bilinear form associated to $u$. By definition of $\|\varphi\|_{c b}$ we have

$$
\left\|\varphi_{N}\left(S_{a}^{(N)}, T_{b}^{(N)}\right)\right\| \leq\|\varphi\|_{c b}\left\|S_{a}^{(N)}\right\|_{M_{N}(E)}\left\|T_{b}^{(N)}\right\|_{M_{N}(E)}
$$

and hence

$$
\limsup _{N \rightarrow \infty}\left\|\varphi_{N}\left(S_{a}^{(N)}, T_{b}^{(N)}\right)\right\| \leq\|\varphi\|_{c b} C_{E}\|a\|_{R C} C_{F}\|b\|_{R C}
$$

But now

$$
\varphi_{N}\left(S_{a}^{(N)}, T_{b}^{(N)}\right)=\sum_{i, j} \varphi\left(a_{i}, b_{j}\right) Y_{i}^{(N)} \otimes \overline{Y_{j}^{(N)}} \in M_{N} \otimes M_{N}=B\left(\ell_{2}^{N} \otimes \ell_{2}^{N}\right)
$$

and the linear form $\psi: M_{N} \otimes M_{N} \rightarrow \mathbb{C}$ defined by $\psi(x \otimes y)=\tau_{N}\left(x^{t} y\right)$ has norm 1 on $B\left(\ell_{2}^{N} \otimes \ell_{2}^{N}\right)$ (indeed its action on $B\left(\ell_{2}^{N} \otimes \ell_{2}^{N}\right)$ can be written as taking $T \in B\left(\ell_{2}^{N} \otimes \ell_{2}^{N}\right)$ to $\langle T \xi$, $\xi\rangle$ with $\xi=$ $\left.N^{-1 / 2} \sum e_{j} \otimes e_{j}\right)$. Therefore

$$
\left|\sum_{i, j} \tau_{N}\left(Y_{i}^{(N)} Y_{j}^{(N)^{*}}\right) \varphi\left(a_{i}, b_{j}\right)\right|=\left|\psi\left(\varphi_{N}\left(S_{a}^{(N)}, T_{b}^{(N)}\right)\right)\right| \leq\left|\varphi_{N}\left(S_{a}^{(N)}, T_{b}^{(N)}\right)\right|
$$

and by weak convergence (see (0.7)) we have $\tau_{N}\left(Y_{i}^{(N)} Y_{j}^{(N)^{*}}\right) \rightarrow \delta_{i, j}$ a.s. when $N \rightarrow \infty$ and hence $\sum_{i, j} \tau_{N}\left(Y_{i}^{(N)} Y_{j}^{(N)^{*}}\right) \varphi\left(a_{i}, b_{j}\right) \rightarrow \sum_{j} \varphi\left(a_{j}, b_{j}\right)$ a.s. so that combining (3.2) and (3.3) we obtain the announced (3.1). 
Remark 3.3. Actually the preceding uses only a weakening of complete boundedness called tracial boundedness, see [21, p. 291].

Remark 3.4. When $E, F$ are completely tight, or merely when $M_{N}(E), M_{N}(F)$ are tight it is natural to try to apply Lemma 3.2 to the bilinear form $\varphi_{N}$. The natural analogous assumption is then for any $a_{1}, \cdots, a_{n} \in M_{N}(E)$ and $b_{1}, \cdots, b_{n} \in M_{N}(F)$

$$
\left\|\sum \varphi_{N}\left(a_{j}, b_{j}\right)\right\|_{M_{N} \otimes_{\min } M_{N}} \leq \lambda\|a\|_{R C}\|b\|_{R C},
$$

Equivalently, let $\xi, \eta$ be arbitrary in the unit ball of $\ell_{2}^{N} \otimes_{2} \ell_{2}^{N}$ and let us denote by $\psi_{\xi, \eta}: M_{N} \otimes_{\min }$ $M_{N} \rightarrow \mathbb{C}$ the linear form defined by $\psi_{\xi, \eta}(T)=\langle T \xi, \eta\rangle$. Note that $\|T\|=\sup \left\{\left|\psi_{\xi, \eta}(T)\right| \mid\|\xi\|_{2} \leq\right.$ 1, $\left.\|\eta\|_{2} \leq 1\right\}$. Therefore, assuming (3.4) is the same as assuming that all the scalar valued bilinear forms $\psi_{\xi, \eta} \varphi_{N}=\psi_{\xi, \eta} \otimes \varphi: M_{N}(E) \times M_{N}(F) \rightarrow \mathbb{C}\left(\right.$ with $\left.\|\xi\|_{2} \leq 1,\|\eta\|_{2} \leq 1\right)$ all satisfy (3.1). Moreover we have

$$
\left\|\varphi_{N}\right\|=\sup \left\{\left\|\psi_{\xi, \eta} \otimes \varphi\right\| \mid\|\xi\|_{2} \leq 1,\|\eta\|_{2} \leq 1\right\} .
$$

The following statement generalizes the main result of [26]. This new formulation became clear after [11] appeared. Indeed, although [11] does not consider it, Mikael de la Salle and the author (see the second proof given in [23, $§ 18$ p. 303]) adapted their method to prove essentially the same as the next result. However, more recently Regev and Vidick gave a strikingly simple proof of the same step. We recommend their paper [27] to the interested reader.

Theorem 3.5. Let $E, F$ be arbitrary operator spaces, let $\varphi: E \times F \rightarrow \mathbb{C}$ be a bilinear form (associated to $u: E \rightarrow F^{*}$ ), and let $\lambda>0$ be any constant. Assume that $\varphi_{N}$ satisfies (3.4) for all $N \geq 1$. Then for any $a_{1}, \cdots, a_{n} \in E$ and $b_{1}, \cdots, b_{n} \in F$ and $t_{1}>0, \cdots, t_{n}>0$

$$
\left|\sum\left\langle u\left(a_{j}\right), b_{j}\right\rangle\right| \leq \lambda\left(\|a\|_{R}\|b\|_{C}+\left\|\left(t_{j} a_{j}\right)\right\|_{C}\left\|\left(t_{j}{ }^{-1} b_{j}\right)\right\|_{R}\right) .
$$

Remark 3.6. Actually, this still holds if the assumption (3.4) imposed on $\varphi_{N}$ is weakened to

$$
\left\|\sum \varphi_{N}\left(a_{j}, b_{j}\right)\right\|_{M_{N} \otimes_{\min } M_{N}} \leq \lambda\left(\|a\|_{R}^{2}+\|a\|_{C}^{2}\right)^{1 / 2}\left(\|b\|_{R}^{2}+\|b\|_{C}^{2}\right)^{1 / 2} .
$$

When $E, F$ are $C^{*}$-algebras, (3.7) and hence (3.6) is satisfied with $\lambda=\|u\|_{c b}$ and this is sharp (see [11] or [23] for details).

Corollary 3.7. If two operator spaces $E, F$ are respectively completely $\hat{C}_{E}$-tight and completely $\hat{C}_{F}$-tight, (for some constants $\hat{C}_{E}, \hat{C}_{F}$ ) then any $u \in C B\left(E, F^{*}\right)$ satisfies for any $a_{1}, \cdots, a_{n} \in E$ and $b_{1}, \cdots, b_{n} \in F$ and $t_{1}>0, \cdots, t_{n}>0$

$$
\left|\sum\left\langle u\left(a_{j}\right), b_{j}\right\rangle\right| \leq \hat{C}_{E} \hat{C}_{F}\|u\|_{c b}\left(\|a\|_{R}\|b\|_{C}+\left\|\left(t_{j} a_{j}\right)\right\|_{C}\left\|\left(t_{j}{ }^{-1} b_{j}\right)\right\|_{R}\right) .
$$

Proof. Let $\varphi: E \times F \rightarrow \mathbb{C}$ be the bilinear form associated to $u$. Let $N \geq 1$. We will invoke Remark 3.4 and (3.5). Let $\lambda=\hat{C}_{E} \hat{C}_{F}\|u\|_{c b}$. Since $M_{N}(E), M_{N}(F)$ are tight, by Lemma 3.2 the mappings $\psi_{\xi, \eta} \otimes \varphi: M_{N}(E) \times M_{N}(F) \rightarrow \mathbb{C}$ all satisfy (3.1) and hence (see Remark 3.4) $\varphi_{N}$ satisfies (3.4), and the Corollary follows from the preceding Theorem.

By the same method as in [26] (see [23, Prop. 18.2]), this implies

Corollary 3.8. If E, $F$ are respectively completely $\hat{C}_{E}$-tight and completely $\hat{C}_{F}$-tight, then any $u \in C B\left(E, F^{*}\right)$ admits, for some Hilbert spaces $H, K$, a factorization of the form

$$
E \stackrel{v}{\longrightarrow} H_{r} \oplus K_{c} \stackrel{w}{\longrightarrow} F^{*}
$$

with $\|v\|_{c b}\|w\|_{c b} \leq 2 \hat{C}_{E} \hat{C}_{F}\|u\|_{c b}$. Here the spaces $K_{c}=B(\mathbb{C}, K)$ and $H_{r}=B(\bar{H}, \mathbb{C})$ are equipped with their natural operator space structure and the direct sum $H_{r} \oplus K_{c}$ is taken in the block diagonal sense. When $H=\ell_{2}$ (resp. $\left.K=\ell_{2}\right)$ we have $H_{r}=R$ (resp. $\left.K_{c}=C\right)$. 


\section{Subexponential operator spaces}

Let $E$ be a finite dimensional operator space. Fix $C>0$. We denote by $K_{E}(N, C)$ the smallest integer $K$ such that there is an operator subspace $F \subset M_{K}$ such that

$$
d_{N}(E, F) \leq C
$$

We will say that an operator space $X$ is $C$-exact if for any finite dimensional subspace $E \subset X$ there is a $K$ and $F \subset M_{K}$ such that $d_{c b}(E, F) \leq C$. We denote by $e x(X)$ the infimum of such $C$ 's. We say that $X$ is exact if it is $C$-exact for some $C \geq 1$. As shown by Kirchberg, a $C^{*}$-algebra $X$ is exact iff $e x(X)=1$. We do not know whether the analogue of this for subexponential $C^{*}$-algebras is true. See [21, ch.17] or [1] for more background on exactness (note however that our definition of $C$-exact is not quite the same as in [21] where $C$-exact means $\operatorname{ex}(X) \leq C)$.

Lemma 4.1. An operator space $X$ is $C$-exact iff

$$
\sup _{N \geq 1} K_{E}(N, C)<\infty .
$$

for any finite dimensional subspace $E \subset X$.

Proof. The only if part is obvious since $d_{N} \leq d_{c b}$. Conversely, assume that for some fixed $K$ we have

$$
\sup _{N \geq 1} K_{E}(N, C) \leq K .
$$

We have then for each $N$ a subspace $F_{N} \subset M_{K}$ and a mapping $u(N): \quad E \rightarrow F_{N}$ such that $\left\|u(N)_{N}\right\| \leq C$ and $\left\|u(N)^{-1}{ }_{N}\right\| \leq 1$. Let $F$ be an ultraproduct of $\left(F_{N}\right)$ along a free ultrafilter (see e.g. 21] for ultraproducts of operator spaces), and let $u: E \rightarrow F$ be the mapping associated to $(u(N))$. Then clearly $\|u\|_{c b} \leq C$ and $\left\|u^{-1}\right\|_{c b} \leq 1$. So we obtain $d_{c b}(E, F) \leq C$ and $F$ obviously embeds completely isometrically into $M_{K}$.

Definition 4.2. We say that an operator space $X$ is $C$-subexponential if

$$
\limsup _{N \rightarrow \infty} \frac{\log K_{E}(N, C)}{N}=0,
$$

for any finite dimensional subspace $E \subset X$. We say that $X$ is subexponential if it is $C$-subexponential for some $C \geq 1$.

Note: If $X$ itself is finite dimensional, it suffices to consider $E=X$.

We will denote by $C(X)$ the infimum of the $C$ 's such that $X$ is $C$-subexponential.

In [25] we introduce the following variant of $K_{E}(N, C)$ :

We denote by $k_{E}(N, C)$ the smallest integer $k$ such that there is a subspace $F$ of $M_{N} \oplus \cdots \oplus M_{N}$ (with $M_{N}$ repeated $k$-times) such that $d_{N}(E, F) \leq C$. Obviously we have

$$
K_{E}(N, C) \leq N k_{E}(N, C)
$$

We observe in [25] that for any $E$ we have

$$
k_{E}(N, C) \leq\left(\frac{3 C}{C-1}\right)^{2 n N^{2}} .
$$


The proof is an easy argument involving the cardinal of a $(C-1)$-net in the unit ball of the space $M_{N}(E)$, the $\mathbb{R}$-dimension of which is $2 n N^{2}$.

This implies that

$$
\frac{\log K_{E}(N, C)}{n N^{2}} \leq C^{\prime}
$$

where $C^{\prime}$ depends only on $C>1$.

Unfortunately, we are unable to improve the last bound for general $n$-dimensional spaces $E$. More precisely, we do not know whether there exists spaces $E$ for which the growth of $\log K_{E}(N, C)$ is intermediate between $o(N)$ (i.e. the subexponential case) and $O\left(N^{2}\right)$ (the general case).

Remark 4.3. It is easy to check that if $X$ is $C$-subexponential, the minimal tensor product $K\left(\ell_{2}\right) \otimes_{\min }$ $X$ (of $X$ with the set $K\left(\ell_{2}\right)$ of all compact operators on $\ell_{2}$ ) is also $C$-subexponential. Indeed, by a perturbation argument we may restrict to finite dimensional subspaces of the form $M_{n}(E)$ with $E \subset X$. Then we have obviously

$$
K_{M_{n}(E)}(N, C) \leq n K_{E}(n N, C),
$$

and hence the subexponential character is preserved.

The following result follows from the main estimates in $\S 2$ in [8], but we take advantage of [2] to formulate an improved inequality for which we can give a quick sketch of proof (inspired from ideas in [8] and [2]).

Theorem 4.4 ([8]). Let $p \geq 2$ be any even integer. For any $a=\left(a_{1}, \cdots, a_{n}\right) \in B(H)^{n}$ such that $\operatorname{tr}\left|a_{j}\right|^{p}<\infty$ for $1 \leq j \leq n$, let $S_{a}=\sum_{1}^{n} Y_{j}^{(N)} \otimes a_{j}$. We have

$$
\mathbb{E}\left(\operatorname{tr} \times \tau_{N}\right)\left|S_{a}\right|^{p} \leq \mathbb{E} \tau_{N}\left|Y^{(N)}\right|^{p} \max \left\{\operatorname{tr}\left(\sum a_{j}^{*} a_{j}\right)^{p / 2}, \operatorname{tr}\left(\sum a_{j} a_{j}^{*}\right)^{p / 2}\right\} .
$$

Proof. Let $p \geq 2$ be any even integer. Let $P_{2}(p)$ denote the set of all partitions of $[1, \ldots, p]$ into subsets each with exactly 2 elements. So an element $\nu$ in $P_{2}$ can be described as a collection of disjoint (unordered) pairs $\left\{k_{j}, \ell_{j}\right\}(1 \leq j \leq n)$ with $k_{j} \neq \ell_{j}$ such that $\{1, \ldots, 2 n\}=\left\{k_{1}, \ldots, k_{n}, \ell_{1}, \ldots, \ell_{n}\right\}$. Let $X=\left(X_{j}\right)(1 \leq j \leq p)$ be a Gaussian sequence of real valued random variables (i.e. all their linear combinations are Gaussian). We first recall the classical Wick formula:

$$
\mathbb{E}\left(X_{1} \cdots X_{p}\right)=\sum_{\nu \in P_{2}(p)} \prod\left\langle X_{k_{j}}, X_{\ell_{j}}\right\rangle
$$

where the product runs over all the blocks $\left\{k_{j}, \ell_{j}\right\}(j=1 \cdots p / 2)$ of $\nu$, and the scalar products are meant in $L_{2}$.

To lighten the notation we set $x_{j}=Y_{j}^{(N)}$. Then, if one develops the product and the trace, it is not hard to deduce from the Wick formula that there is a function $\psi: P_{2}(p) \rightarrow \mathbb{C}$ such that for any $k_{1}, \ldots, k_{p}$ we have

$$
\mathbb{E} \tau_{N}\left(x_{k_{1}}^{*} x_{k_{2}} x_{k_{3}} \ldots x_{k_{p-1}}^{*} x_{k_{p}}\right)=\sum_{\nu \sim\left(k_{1}, \ldots, k_{p}\right)} \psi(\nu)
$$

where the notation $\nu \sim\left(k_{1}, \ldots, k_{p}\right)$ means that $k_{i}=k_{j}$ whenever the pair $\{i, j\}$ is a block of the partition $\nu$.

Note that, for each $k$, taking the $k_{j}$ 's all equal to $k$, this implies

$$
\mathbb{E} \tau_{N}\left(\left|x_{k}\right|^{p}\right)=\sum_{\nu \in P_{2}(p)} \psi(\nu)
$$


We may complete the proof without spelling out the precise formula for $\psi(\nu)$ but we need to note that $\psi(\nu) \geq 0$ and that the only $\nu$ 's for which $\psi(\nu) \neq 0$ are those with all blocks formed of an odd and an even index. We have $\left|S_{a}\right|^{p}=\left(S_{a}^{*} S_{a}\right)^{p / 2}$ and hence

$$
\mathbb{E}\left(\operatorname{tr} \times \tau_{N}\right)\left|S_{a}\right|^{p}=\sum_{k_{1}, \ldots, k_{p}} \sum_{\nu \sim\left(k_{1}, \ldots, k_{p}\right)} \psi(\nu) \operatorname{tr}\left(a_{k_{1}}^{*} a_{k_{2}} \cdots a_{k_{p-1}}^{*} a_{k_{p}}\right) .
$$

Therefore

$$
\mathbb{E}\left(\operatorname{tr} \times \tau_{N}\right)\left|S_{a}\right|^{p} \leq \sum_{\nu \in P_{2}(p)} \psi(\nu) \operatorname{tr}\left(a_{\nu}\right) \leq\left(\sum_{\nu \in P_{2}(p)} \psi(\nu)\right) \max _{\nu} \operatorname{tr}\left(a_{\nu}\right)
$$

where $a_{\nu}=\sum_{\left(k_{1}, \ldots, k_{p}\right) \sim \nu}\left(a_{k_{1}}^{*} a_{k_{2}} \cdots a_{k_{p-1}}^{*} a_{k_{p}}\right)$. Now by a nice iteration argument of the CauchySchwarz inequality, for which the reader can find details in [2], one can show that the terms $\operatorname{tr}\left(a_{\nu}\right)$ are maximal when $\nu$ is either $\{1,2\},\{3,4\} \cdots\{p-1, p\}$ or $\{p, 1\},\{2,3\} \cdots\{p-2, p-1\}$ (i.e. a partition in cyclically consecutive pairs), in which case by the trace property we have either $\operatorname{tr}\left(a_{\nu}\right)=\operatorname{tr}\left(\sum a_{j}^{*} a_{j}\right)^{p / 2}$ or $\operatorname{tr}\left(a_{\nu}\right)=\operatorname{tr}\left(\sum a_{j} a_{j}^{*}\right)^{p / 2}$. Thus, by (4.4) we obtain (4.3).

Note that (4.3) is obviously best possible. It can be interpreted as a sort of "Khintchine inequality" for Gaussian random matrices with best possible constant.

We will use the following direct consequence of Theorem 4.4 and concentration of measure.

Corollary 4.5. For any $\varepsilon>0$, there is a constant $\gamma_{\varepsilon}$ such that for any integer $k$ and any $a_{1}, \cdots, a_{n} \in M_{k}$ we have

$$
\mathbb{E}\left\|\sum_{1}^{n} Y_{j}^{(N)} \otimes a_{j}\right\| \leq(1+\varepsilon)\left(2+\gamma_{\varepsilon}\left(\frac{\log (k)+1}{N}\right)^{1 / 2}\right) \max \left\{\left\|\left(\sum a_{j}^{*} a_{j}\right)^{1 / 2}\right\|,\left\|\left(\sum a_{j} a_{j}^{*}\right)^{1 / 2}\right\|\right\} .
$$

Proof. Let $X$ be any Gaussian random variable with values in a (real) Banach space B. Let

$$
\sigma(X)=\sup \left\{\left(\mathbb{E}|\xi(X)|^{2}\right)^{1 / 2} \mid \xi \in B^{*},\|\xi\| \leq 1\right\}
$$

It will be convenient to use the following concentration of measure inequality (see [18] for a very simple proof):

$$
\|\| X\|-\mathbb{E}\| X\|\|_{p} \leq(\pi / 2) \sigma(X)\|g\|_{p},
$$

where $g$ is a standard Gaussian normal random variable, and this implies

$$
\left(\mathbb{E}\|X\|^{p}\right)^{1 / p} \leq \mathbb{E}\|X\|+(\pi / 2) \sigma(X)\|g\|_{p} .
$$

We will view $Y^{(N)}$ as $B$-valued with $B=M_{N}$ considered as a real Banach space. We have then

$$
\sigma\left(Y^{(N)}\right) \leq N^{-1 / 2}
$$

We denote $\left\|Y^{(N)}\right\|_{p}=\left(\operatorname{tr}\left(\left|Y^{(N)}\right|^{p}\right)^{1 / p}\right.$. Thus the preceding inequality applied to $X=Y^{(N)}$ yields by (4.6)

$$
\left(\mathbb{E}\left\|Y^{(N)}\right\|_{p}^{p}\right)^{1 / p} \leq(N)^{1 / p}\left(\mathbb{E}\left\|Y^{(N)}\right\|^{p}\right)^{1 / p} \leq(N)^{1 / p}\left(\mathbb{E}\left\|Y^{(N)}\right\|+(\pi / 2) \sigma\left(Y^{(N)}\right)\|g\|_{p}\right) .
$$

It is well known that $\lim _{N \rightarrow \infty} \mathbb{E}\left\|Y^{(N)}\right\|_{M_{N}}=2$. In fact we need only an upper bound, so we set

$$
\varepsilon(N)=\mathbb{E}\left\|Y^{(N)}\right\|-2 \text { and we note } \lim _{N \rightarrow \infty} \varepsilon(N)=0 .
$$

Since there is a constant $\beta$ such that $\|g\|_{p} \leq \beta \sqrt{p}$ for all $p \geq 1$, we find

$$
\left(\mathbb{E}\left\|Y^{(N)}\right\|_{p}^{p}\right)^{1 / p} \leq(N)^{1 / p}\left(2+\varepsilon(N)+\beta(\pi / 2)(p / N)^{1 / 2}\right)
$$


Let $S=S_{a}$. We again denote $\|S\|_{p}=\left(\operatorname{tr}\left(|S|^{p}\right)^{1 / p}\right.$ (but this time the trace is on $\left.M_{N} \otimes M_{k}\right)$. We have obviously $\|S\| \leq\|S\|_{p}$ for any $p \geq 1$ and hence

$$
\left(\mathbb{E}\|S\|^{p}\right)^{1 / p} \leq\left(\mathbb{E}\|S\|_{p}^{p}\right)^{1 / p} .
$$

By homogeneity we may assume $\max \left\{\left\|\left(\sum a_{j}^{*} a_{j}\right)^{1 / 2}\right\|,\left\|\left(\sum a_{j} a_{j}^{*}\right)^{1 / 2}\right\|\right\} \leq 1$. By (4.3) this gives us

$$
\left(\mathbb{E}\|S\|^{p}\right)^{1 / p} \leq(k N)^{1 / p}\left(2+\varepsilon(N)+\beta(\pi / 2)(p / N)^{1 / 2}\right) .
$$

Fix $0<\varepsilon \leq 1$. For a suitably chosen $N(\varepsilon)$, we have for all $N \geq N(\varepsilon)$

$$
\left(\mathbb{E}\|S\|^{p}\right)^{1 / p} \leq(k N)^{1 / p}\left(2+\varepsilon / 2+\beta(\pi / 2)(p / N)^{1 / 2}\right) .
$$

We can choose the even integer $p$ large enough so that $(k N)^{1 / p} \approx 1+\varepsilon / 2$ : Indeed, by taking say $p=2\left[c \varepsilon^{-1}(\log (k N)+1)\right]$ and adjusting the positive constant $c$ and $N(\varepsilon)$ we can obtain $(k N)^{1 / p} \leq$ $1+\varepsilon / 2$. Then, for some numerical constant $\beta^{\prime}$, we obtain

$$
\mathbb{E}\|S\| \leq\left(\mathbb{E}\|S\|^{p}\right)^{1 / p} \leq(1+\varepsilon / 2)\left(2+\varepsilon / 2+\beta^{\prime}\left(\varepsilon^{-1} \log (k N) / N\right)^{1 / 2}\right),
$$

and this leads to (4.5), at least for all $N \geq N(\varepsilon)$, with $N(\varepsilon)$ depending only on $\varepsilon$.

But for $N \leq N(\varepsilon)$ it is easy to choose the constant $\gamma_{\varepsilon} \geq N(\varepsilon)$ to make sure that (4.5) remains true. Indeed, for some $\beta_{\varepsilon}$ we have $\varepsilon(N) \leq \beta_{\varepsilon}$ for all $N \geq N(\varepsilon)$.

Remark 4.6. By [8, Th. 3.3], with the same notation as in the above Theorem 4.4, assuming $\max \left\{\left\|\left(\sum a_{j}^{*} a_{j}\right)^{1 / 2}\right\|,\left\|\left(\sum a_{j} a_{j}^{*}\right)^{1 / 2}\right\|\right\} \leq 1$ we have for any $0 \leq t \leq N / 2$

$$
\mathbb{E} \exp t\|S\|^{2} \leq k N \exp \left(4 t+4 t^{2} / N\right) .
$$

By convexity this implies $\exp t(\mathbb{E}\|S\|)^{2} \leq k N \exp \left(4 t+4 t^{2} / N\right)$, and hence taking the log we find

$$
\mathbb{E}\|S\| \leq 2\left(1+t / N+(4 t)^{-1} \log (k N)\right)^{1 / 2}
$$

from which taking $t=[\varepsilon N]$ for $\varepsilon<1 / 2$ it is easy to deduce (4.5). Note however that the deduction of [8, Th. 3.3] from Prop. 2.5 and Prop. 2.7 in [8] involves rather heavy calculations, and that explains why we presented the above short cut (based only on Prop. 2.5 and Prop. 2.7 in [8]) using concentration of measure instead of invoking [8, Th. 3.3]. In addition, this route allows us to draw the reader's attention to Buchholz's nice contribution [2].

Corollary 4.7. For any finite dimensional operator space $E$ and any $a_{1}, \cdots, a_{n} \in E$ we have

$\mathbb{E}\left\|\sum_{1}^{n} Y_{j}^{(N)} \otimes a_{j}\right\| \leq C(1+\varepsilon)\left(2+\gamma_{\varepsilon}\left(\frac{\log \left(K_{E}(N, C)\right)+1}{N}\right)^{1 / 2}\right) \max \left\{\left\|\left(\sum a_{j}^{*} a_{j}\right)^{1 / 2}\right\|,\left\|\left(\sum a_{j} a_{j}^{*}\right)^{1 / 2}\right\|\right\}$.

Proof. Consider $u: E \rightarrow F$ with $F \subset M_{k}, k=K_{E}(N, C)$ and $\left\|u_{N}\right\|\left\|u^{-1}{ }_{N}\right\| \leq C$.

By homogeneity we may assume $\max \left\{\left\|\left(\sum a_{j}^{*} a_{j}\right)^{1 / 2}\right\|,\left\|\left(\sum a_{j} a_{j}^{*}\right)^{1 / 2}\right\|\right\}=1$. Let $b_{j}=u\left(a_{j}\right)$. We may assume $n \leq N$. Then we have

$$
\max \left\{\left\|\left(\sum b_{j}^{*} b_{j}\right)^{1 / 2}\right\|,\left\|\left(\sum b_{j} b_{j}^{*}\right)^{1 / 2}\right\|\right\} \leq\left\|u_{n}\right\| \leq\left\|u_{N}\right\|,
$$

and also

$$
\left\|\sum_{1}^{n} Y_{j}^{(N)} \otimes a_{j}\right\| \leq\left\|u^{-1}{ }_{N}\right\|\left\|\sum_{1}^{n} Y_{j}^{(N)} \otimes b_{j}\right\| .
$$


By (4.5) (applied with $b_{j}$ in place of $a_{j}$ ) this gives us

$$
\mathbb{E}\left\|\sum_{1}^{n} Y_{j}^{(N)} \otimes a_{j}\right\| \leq\left\|u^{-1}{ }_{N}\right\|\left\|u_{N}\right\|(1+\varepsilon)\left(2+\gamma_{\varepsilon}\left(\frac{\log (k)+1}{N}\right)^{1 / 2}\right),
$$

and the result follows.

This leads us immediately to

Theorem 4.8. Any C-subexponential operator space is completely $2 C$-tight.

Proof. By Remark 4.3 it suffices to show that $E$ is $2 C$-tight. Then, letting $N \rightarrow \infty$, the result follows from the preceding Corollary.

For emphasis, we state the following immediate consequence of Corollary 3.8 ,

Corollary 4.9. If $E, F$ are both subexponential, then any $u \in C B\left(E, F^{*}\right)$ admits, for some Hilbert spaces $H, K$, a factorization of the form

$$
E \stackrel{v}{\longrightarrow} H_{r} \oplus K_{c} \stackrel{w}{\longrightarrow} F^{*}
$$

with $\|v\|_{c b}\|w\|_{c b} \leq 4 C(E) C(F)$.

Using Oikhberg's result as in [23, p. 296] we obtain

Corollary 4.10. If $E, E^{*}$ are both subexponential, then for some Hilbert spaces $H, K, E$ must be completely isomorphic to $H_{r} \oplus K_{c}$. If $E$ is separable and infinite dimensional then $E$ must be completely isomorphic to either $R, C$ or $R \oplus C$.

Remark 4.11. Perhaps the preceding statement is best appreciated for a finite dimensional space $E$. Roughly if $E$ is not close to a space of the form $H_{r} \oplus K_{c}$ (which in practise is easy to see since very few spaces are like that), then either $K_{E}(N, C)$ or $K_{E^{*}}(N, C)$ must grow superexponentially. This can be viewed as analogous to the Figiel-Lindenstrauss-Milman estimate of the number of faces and vertices of a polytope in [5, Th. 3.4].

Remark 4.12. The preceding proofs suggest that perhaps one should keep track of the dependence in $E$ in studying spaces like subexponential ones. One possibility would be to define $X$ as $\left(C, C^{\prime}\right)$ subexponential if for any finite dimensional $E \subset X$ we have

$$
\limsup _{N \rightarrow \infty} N^{-1} \log K_{E}(N, C) \leq C^{\prime} .
$$

Note however that the constant $C^{\prime}$ does not seem to behave as well as $C$ (see (4.2)) when one passes from $E$ to $M_{n}(E)$.

Remark 4.13. Given an operator space $X$, it is natural to introduce the following parameter:

$$
K_{X}(N, C ; d)=\sup \left\{K_{E}(N, C) \mid E \subset X, \operatorname{dim}(E)=d\right\} .
$$

We will say that $X$ is uniformly subexponential if there is $C$ such that

$$
\forall d \geq 1 \quad \limsup _{N \rightarrow \infty} \frac{\log K_{X}(N, C ; d)}{N}=0 .
$$

Similarly we will say that $X$ is uniformly exact if there is $C$ such that

$$
\forall d \geq 1 \quad \sup \left\{K_{X}(N, C ; d) \mid N \geq 1\right\}<\infty .
$$


It is easy to check that if $X$ is uniformly exact (resp. uniformly subexponential) then all ultrapowers of $X$ are exact (resp. subexponential). Note however (I am indebted to Yanqi Qiu for conversations on this) that the converse is unclear.

For example, $R$ or $C$ and $R \oplus C$ are uniformly exact. More generally, let $A$ (resp. $(\Omega, \mu)$ ) be any commutative $C^{*}$ algebra (resp. any measure space), then $A$ (or $L_{\infty}(\Omega, \mu)$ ) and any space of the form $A \otimes_{\min } M_{N}$ (with $N$ fixed) or $L_{\infty}(\Omega, \mu ; R \oplus C)$ is uniformly exact. There seem to be rather few such spaces. It would be interesting to characterize them.

Remark 4.14. It is tempting to weaken the definition of subexponential spaces by replacing the limsup there by a liminf. Such spaces could be called weakly subexponential. We do not know whether this is a true weakening. Then Corollary 4.9 extends to the case when one of $E, F$ is weakly subexponential and the other one subexponential. Note however that, a priori, the case when both $E, F$ are weakly subexponential is unclear.

\section{Large constants of subexponentiality}

We will now examine some examples. It turns out that the most commonly known non-exact operator spaces are also not subexponential, and the associated constants have a similar growth.

We start by discussing maximal operator spaces. (See e.g. [21] for the definitions of minimal and maximal operator spaces.)

Proposition 5.1. Let $E$ be any n-dimensional space with its maximal operator space structure. Then

$$
C(E) \geq c \sqrt{n}
$$

where $c>0$ is a constant independent of $n$.

Proof. We transplant from exact to subexponential an argument from [12. Note that $C\left(E^{*}\right)=1$ since $E^{*}$ is a minimal operator space. By Theorem 4.8 and Lemma 3.2 (with $F^{*}=E$ and $u$ the identity of $E$ ) we have for all finite sequences $\left(a_{j}, b_{j}\right)$ in $E \times E^{*}$

$$
\left|\sum\left\langle a_{j}, b_{j}\right\rangle\right| \leq 4 C(E)\|a\|_{R C}\|b\|_{R C}
$$

but here $\|b\|_{R C}=\sup \left\{\left(\sum\left|b_{j}(x)\right|^{2}\right)^{1 / 2} \mid x \in E,\|x\| \leq 1\right\}$ and $\|a\|_{R C} \leq\left(\sum\left\|a_{j}\right\|^{2}\right)^{1 / 2}$, so this implies

$$
\left|\sum\left\langle a_{j}, b_{j}\right\rangle\right| \leq 4 C(E)\left(\sum\left\|a_{j}\right\|^{2}\right)^{1 / 2} \sup \left\{\left(\sum\left|b_{j}(x)\right|^{2}\right)^{1 / 2} \mid x \in E,\|x\| \leq 1\right\}
$$

and hence

$$
\left(\sum\left\|b_{j}\right\|^{2}\right)^{1 / 2} \leq 4 C(E) \sup \left\{\left(\sum\left|b_{j}(x)\right|^{2}\right)^{1 / 2} \mid x \in E,\|x\| \leq 1\right\} .
$$

Equivalently, this means the 2-summing norm $\pi_{2}(E)$ of the identity of $E$ is $\leq 4 C(E)$. But it is well known (see e.g. [19, p. 35]) that $\pi_{2}(E)=\sqrt{n}$. Thus we conclude $C(E) \geq \sqrt{n} / 4$.

Remark 5.2. In the converse direction, for any $n$-dimensional operator space $E$ we have $C(E) \leq$ $e x(E)$ and it is known (see [21, Cor. 7.7 p. 133]) that $e x(E) \leq \sqrt{n}$.

Remark 5.3. We claim that

$$
n^{1 / 4} / 2 \leq C\left(O H_{n}\right) \leq n^{1 / 4} .
$$

Indeed, applying Corollary 4.7 with $E=O H_{n}$ (see [21, $\left.\S 7\right]$ ) and with $a_{j}$ an orthonormal basis we find

$$
\limsup _{N \rightarrow \infty} \mathbb{E}\left(\left\|\sum_{1}^{n} Y_{j}^{(N)} \otimes \overline{Y_{j}^{(N)}}\right\|^{1 / 2}\right) \leq 2 C\left(O H_{n}\right) n^{1 / 4},
$$


and since $\left\|\sum_{1}^{n} Y_{j}^{(N)} \otimes \overline{Y_{j}^{(N)}}\right\| \geq \sum_{1}^{n} \tau_{N}\left|Y_{j}^{(N)}\right|^{2}$ and (by the law of large numbers) $\sum_{1}^{n} \tau_{N}\left|Y_{j}^{(N)}\right|^{2} \approx n$ we obtain

$$
n^{1 / 2}=\limsup _{N \rightarrow \infty} \mathbb{E}\left(\left(\sum_{1}^{n} \tau_{N}\left|Y_{j}^{(N)}\right|^{2}\right)^{1 / 2}\right) \leq 2 C\left(O H_{n}\right) n^{1 / 4}
$$

and hence $C\left(O H_{n}\right) \geq n^{1 / 4} / 2$. In the converse direction, we have $C\left(O H_{n}\right) \leq e x\left(O H_{n}\right)$ and it is known (see [21, (10.8) p. 219]) that ex $\left(O H_{n}\right) \leq n^{1 / 4}$.

Remark 5.4. We claim that

$$
n^{1 / 2} / 2 \leq C\left(R_{n}+C_{n}\right) \leq n^{1 / 2} .
$$

Indeed, applying Corollary 4.7 with $E=R_{n}+C_{n}$ (see [21, §2.7]) and with $a_{j}$ an orthonormal basis we find similarly (since $\left\|\left(a_{j}\right)\right\|_{R C}=1$ )

$$
n^{1 / 2}=\limsup _{N \rightarrow \infty} \mathbb{E}\left(\left(\sum_{1}^{n} \tau_{N}\left|Y_{j}^{(N)}\right|^{2}\right)^{1 / 2}\right) \leq 2 C\left(R_{n}+C_{n}\right),
$$

and thus we obtain

$$
C\left(R_{n}+C_{n}\right) n^{-1 / 2} \geq 1 / 2 .
$$

In the converse direction, by Remark 5.2 we have $C\left(R_{n}+C_{n}\right) \leq n^{1 / 2}$.

\section{More growth estimates}

In [17] the parameter denoted below by $n(E, c)$ was introduced for an $n$-dimensional Banach space $E$ and a constant $c$ (in [17] we fixed $c=2$ ). We denote by $n(E, c)$ the smallest $k$ such that $E$ can be embedded $c$-isomorphically into $\ell_{\infty}^{k}$. In Banach space theory Gaussian random variables can be used to give a quick proof of the fact that if either $E=\ell_{2}^{n}$ or $E=\ell_{1}^{n}$ then there is $\delta=\delta_{c}>0$ such that $n(E, c) \geq \exp (\delta n)$. In [17] an estimate due to Maurey is presented showing that this superexponential behaviour remains true (with $\delta=\delta\left(c, c^{\prime}\right)>0$ ) whenever $E^{*}$ has type $p>1$ with constant at most $c^{\prime}$. Incidentally, it remains an important open question whether this is true assuming only that $E$ has cotype $q<\infty$ with constant at most $c^{\prime}$.

The problem of estimating the number $k_{E}(N, C)$ (as defined just before (4.1)) is entirely analogous to the one considered in [17] for $n(E, c)$. More precisely, we have simply $n(E, C)=k_{E}(1, C)$.

The preceding inequality (4.5) allows us, in the next Lemma, to prove analogous results, for some operator spaces. Note that in the Banach space case (equivalently in the case $N=1$ ), and taking say $c=2$, we also know that $n(E, 2) \leq \exp \left(\delta^{\prime} n\right)$ for some universal constant $\delta^{\prime}$, while (4.1) tells us that for any $n$-dimensional operator space $E$ we have $K_{E}(N, 2) \leq \exp \left(\delta^{\prime} n N^{2}\right)$. Unfortunately we do not know whether this upper bound can be improved to match the lower bound appearing below in (6.1).

Lemma 6.1. If $E$ is $\ell_{1}^{n}$ equipped with its maximal operator space structure, then for any $C>1$ there are an integer $n_{0}$ and $\delta>0$ depending only on $C$ such that for any $n \geq n_{0}, N \geq 1$ we have

$$
K_{E}(N, C) \geq \exp \delta N n
$$

If $E=R_{n}+C_{n}$ or $\ell_{2}^{n}$ equipped with its maximal operator space structure (resp. $E=O H_{n}$ ), this still holds (resp. we have $K_{E}(N, C) \geq \exp \delta N n^{1 / 2}$ ) for all $N \geq n$.

Proof. With the notation in Theorem 4.4, let $a_{j}$ be the canonical basis of $\ell_{1}^{n}$ (resp. $O H_{n}$, rresp. $R_{n}+C_{n}$ ) and let $S=S_{a}$. Then it is easy to check on the one hand that $\mathbb{E}\|S\| \geq \alpha n$ (resp. rresp. 
$\left.\mathbb{E}\|S\| \geq \alpha n^{1 / 2}\right)$ for some $\alpha>0$. On the other hand $\|a\|_{R C}=\max \left\{\left\|\left(\sum a_{j}^{*} a_{j}\right)^{1 / 2}\right\|,\left\|\left(\sum a_{j} a_{j}^{*}\right)^{1 / 2}\right\|\right\}$ is equal to $n^{1 / 2}$ (resp. $n^{1 / 4}$, rresp. 1). Thus by Corollary 4.7 we find if $E=\ell_{1}^{n}$

$$
\alpha n \leq C(1+\varepsilon)\left(2+\gamma_{\varepsilon}\left(\frac{\log \left(K_{E}(N, C)\right)+1}{N}\right)^{1 / 2}\right) n^{1 / 2},
$$

from which we deduce for $n$ large enough

$$
(\alpha / C(1+\varepsilon)) n^{1 / 2} \approx(\alpha / C(1+\varepsilon)) n^{1 / 2}-2 \leq \gamma_{\varepsilon}\left(\frac{\log \left(K_{E}(N, C)\right)+1}{N}\right)^{1 / 2},
$$

which is the announced lower bound (taking e.g. $\varepsilon=1$ ). The cases $E=O H_{n}$ and $E=R_{n}+C_{n}$ are similar. When $\left(a_{j}\right)$ is the basis of $E=\ell_{2}^{n}$ with its maximal operator space structure, by a well known result (see Exercise 28.1 in [21]) we have also a lower bound $\mathbb{E}\|S\| \geq \alpha n$ provided $n \leq N$. In this case, the remaining estimate of $\|a\|_{R C}$ required to complete the proof can be found in [21, p. 223].

\section{Examples of non exact subexponential $C^{*}$-algebras}

In this section, we will show that the (random) $C^{*}$-algebra generated by the block direct sum of a sequence of i.i.d. random matrices is almost surely subexponential with constant 1 and not exact. We will first isolate, in Theorem 7.2 below, the properties of a deterministic (non random) sequence of block direct sum operators that guarantee that the generated $C^{*}$-algebra has the desired properties. For that purpose, we need some preparation.

Consider the direct sum $B=\oplus_{m \geq 1} M_{m}$. By definition, for any $x=\oplus_{m \geq 1} x(m) \in B$ we have $\|x\|=\sup _{m \geq 1}\|x(m)\|$. We equip $M_{m}$ with its normalized trace $\tau_{m}$.

Let $u_{j}=\oplus_{m} u_{j}(m)$ be elements of $B$. Let $\mathcal{A}$ be the unital $C^{*}$-algebra generated by $u_{1}, u_{2}, \cdots, u_{n}$. For simplicity we set $u_{0}=1$. Let $\mathcal{C}$ be a unital $C^{*}$-algebra that we assume generated by $c_{1}, c_{2}, \cdots$ and equipped with a faithful tracial state $\tau$. We again set $c_{0}=1$.

We say (following [14]) that $\left\{u_{j}(m) \mid 1 \leq j \leq n\right\}$ tends strongly to $\left\{c_{j} \mid 1 \leq j \leq n\right\}$ when $m \rightarrow \infty$ if it tends weakly (meaning "in moments" relative to $\tau_{m}$ and $\tau$ ) and moreover $\left\|P\left(u_{i}(m)\right)\right\| \rightarrow\left\|P\left(c_{i}\right)\right\|$ for any (non-commutative) $*$-polynomial $P$. This implies that for any finite set $P_{0}, P_{1}, \cdots, P_{q}$ of such polynomials, for any $k$ and any $a_{j} \in M_{k}$ we have

$$
\lim _{m \rightarrow \infty}\left\|\sum_{0}^{q} a_{j} \otimes P_{j}\left(u_{i}(m)\right)\right\|=\left\|\sum_{0}^{q} a_{j} \otimes P_{j}\left(c_{i}\right)\right\| .
$$

In particular we have

$$
\lim _{m \rightarrow \infty}\left\|\sum_{0}^{n} a_{j} \otimes u_{j}(m)\right\|=\left\|\sum_{0}^{n} c_{j} \otimes a_{j}\right\| .
$$

Let $I_{0} \subset B$ denote the ideal of sequences $\left(x_{m}\right) \in B$ that tend to zero in norm (usually denoted by $c_{0}\left(\left\{M_{N_{m}}\right\}\right)$. Let $Q: B \rightarrow B / I_{0}$ be the quotient map. It is easy to check that for any polynomial $P$ we have $\left\|Q\left(P\left(u_{j}\right)\right)\right\|=\left\|P\left(c_{j}\right)\right\|$. So that, if we set $I=I_{0} \cap \mathcal{A}$, we have a natural identification

$$
\mathcal{A} / I=\mathcal{C} \text {. }
$$

Let $P_{d}$ denote the linear space of all polynomials of degree $\leq d$ in the non commutative variables $\left(X_{1}, \cdots, X_{n}, X_{1}^{*}, \cdots, X_{n}^{*}\right)$. We will need to consider the space $M_{k} \otimes P_{d}$. It will be convenient to systematically use the following notational convention:

$$
\forall 1 \leq j \leq n \quad X_{n+j}=X_{j}^{*}
$$


A typical element of $M_{k} \otimes P_{d}$ can then be viewed as a polynomial $P=\sum a_{J} \otimes X^{J}$ with coefficients in $M_{k}$. Here the index $J$ runs over the disjoint union of the sets $\{1, \cdots, 2 n\}^{i}$ with $1 \leq i \leq d$. We also add symbolically the value $J=0$ to the index set and we set $X^{0}$ equal to the unit.

We denote by $P(u(m)) \in M_{k} \otimes M_{m}$ (resp. $\left.P(c) \in M_{k} \otimes \mathcal{C}\right)$ the result of substituting $\left\{u_{j}(m)\right\}$ (resp. $\left.\left\{c_{j}\right\}\right)$ in place of $\left\{X_{j}\right\}$. It follows from the strong convergence of $\left\{u_{j} \mid 1 \leq j \leq n\right\}$ to $\left\{c_{j} \mid 1 \leq j \leq n\right\}$ that for any $d$ and any $P \in M_{k} \otimes P_{d}$ we have

$$
\|P(u(m))\| \rightarrow\|P(c)\|
$$

With a similar convention we will write e.g. $P(c)=\sum a_{J} \otimes c^{J}$.

In particular this implies (actually this already follows from weak convergence)

$$
\forall k \forall d \forall P \in M_{k} \otimes P_{d} \quad\|P(c)\| \leq \liminf _{m \rightarrow \infty}\|P(u(m))\| .
$$

Remark 7.1. Let us write $P$ as a sum of monomials $P=\sum a_{J} \otimes X^{J}$ as above. We will assume that the operators $\left\{c^{J}\right\}$ are linearly independent. From this assumption follows that there is a constant $c_{2}(n, d)$ such that

$$
\sum_{J}\left\|a_{J}\right\| \leq c_{2}(n, d)\|P(c)\|
$$

Indeed, since the span of the $c^{J}$ 's is finite dimensional, the linear form that takes $P$ to its $c^{J}$ coefficient is continuous, and its norm (that depends obviously only on $(n, d)$ ) is the same as its c.b. norm. Of course this depends also on the distribution of the family $\left\{c_{j}\right\}$ but we view this as fixed from now on.

We will consider the following assumption:

$$
\sum_{1}^{n} \tau\left(\left|c_{j}\right|^{2}\right)>\left\|\sum_{1}^{n} u_{j} \otimes \bar{c}_{j}\right\|_{\mathcal{A} \otimes_{\min } \overline{\mathcal{C}}} .
$$

Notation. Let $\alpha \subset \mathbb{N}$ be a subset (usually infinite in the sequel). We denote

$$
\begin{gathered}
B(\alpha)=\oplus_{m \in \alpha} M_{m} . \\
u_{j}(\alpha)=\oplus_{m \in \alpha} u_{j}(m) \in B(\alpha) .
\end{gathered}
$$

We will denote by $A(\alpha) \subset B(\alpha)$ the unital $C^{*}$-algebra generated by $\left\{u_{j}(\alpha) \mid 1 \leq j \leq n\right\}$. With this notation $\mathcal{A}=A(\mathbb{N})$ and $u_{j}=u_{j}(\mathbb{N})$.

We also set $E_{d}(\alpha)=\left\{P(u(\alpha)) \mid P \in P_{d}\right\}$.

Fix a degree $d \geq 1$. Then for any real numbers $m \geq 1$ and $t \geq 1$ we define

$$
C_{d}(t, m)=\sup _{m^{\prime} \geq m} \sup _{k \leq t}\left\{\left\|P\left(u\left(m^{\prime}\right)\right)\right\| \mid P \in M_{k} \otimes P_{d},\|P(c)\| \leq 1\right\} .
$$

Theorem 7.2. Assume that for any $d \geq 1$ there are $a>0$ and $D>0$ such that $C_{d}\left(N, a N^{D}\right) \rightarrow 1$ when $N \rightarrow \infty$. Assume moreover that (17.3) holds and that $\mathcal{C}$ is exact. Then for any subset $\alpha \subset \mathbb{N}$ the unital $C^{*}$-algebra $A(\alpha)$ generated by $\left\{u_{j}(\alpha) \mid 1 \leq j \leq n\right\}$ is $1+\varepsilon$-subexponential for any $\varepsilon>0$. Moreover, if we assume that that $\left\{u_{j}(m) \mid 1 \leq j \leq n\right\}$ tends weakly (meaning "in moments" relative to $\tau_{m}$ and $\left.\tau\right)$ to $\left\{c_{j} \mid 1 \leq j \leq n\right\}$ when $m \rightarrow \infty$ and if (17.4) holds then $A(\alpha)$ is not exact. 
Proof. For subexponentiality, we need to show that for any fixed $\varepsilon>0$ and any finite dimensional subspace $E \subset A(\alpha)$ the growth of $N \mapsto K_{E}(N, 1+\varepsilon)$ is subexponential. Since the polynomials in $\left\{u_{j}(\alpha)\right\}$ are dense in $A(\alpha)$, by perturbation it suffices to check this for $E \subset E_{d}(\alpha)$. Thus we may as well assume $E=E_{d}(\alpha)$.

Then we may choose $N_{0}$ large enough so that $C_{d}\left(N, a N^{D}\right)<1+\varepsilon$ for all $N \geq N_{0}$. We claim that for all $N \geq N_{0}$ we have $K_{E}(N, 1+\varepsilon) \in O\left(N^{2 D}\right)$ when $N \rightarrow \infty$. To verify this, let $P \in M_{N} \otimes P_{d}$. Then, recalling (7.3), we have

$$
\|P(c)\| \leq \sup _{m \geq a N^{D}}\|P(u(m))\| \leq C_{d}\left(N, a N^{D}\right)\|P(c)\| .
$$

Let $\alpha^{\prime}=\alpha \cap\left[1, a N^{D}\right)$. Let $T: E \rightarrow B\left(\alpha^{\prime}\right) \oplus \mathcal{C}$ be the linear mapping defined for all $P$ in $P_{d}$ by

$$
T\left(P(u(\alpha))=P\left(u\left(\alpha^{\prime}\right)\right) \oplus P(c) .\right.
$$

We may assume $\alpha$ infinite (otherwise the subexponentiality is trivial). Then (7.3) shows that $\|T\|_{c b} \leq 1$. Conversely, by (7.5) we have

$$
\left\|\left(T^{-1}\right)_{N}\right\| \leq C_{d}\left(N, a N^{D}\right)<1+\varepsilon .
$$

Let $\hat{E}$ be the range of $T$. This shows that $d_{N}(E, \hat{E})<1+\varepsilon$. We have $\hat{E} \subset \oplus_{k<a N^{D}} M_{k} \oplus \hat{E}^{\prime}$ where $\hat{E}^{\prime}$ is a finite dimensional subspace of $\mathcal{C}$ (included in the span of polynomials of degree $d$ ). Since $\mathcal{C}$ is exact, there is an integer $K$ such that, for any $\varepsilon^{\prime}>0, \hat{E}^{\prime}$ is completely $\left(1+\varepsilon^{\prime}\right)$-isomorphic to a subspace of $M_{K}$, so that $\hat{E}$ is completely $\left(1+\varepsilon^{\prime}\right)$-isomorphic to a subspace of $\oplus_{k<a N^{D}} M_{k} \oplus M_{K}$. Therefore, if we choose $\varepsilon^{\prime}$ such that $\left(1+\varepsilon^{\prime}\right) d_{N}(E, \hat{E})<1+\varepsilon$, we have for any $N \geq N_{0}$

$$
K_{E}(N, 1+\varepsilon) \leq 1+2+\cdots+\left[a N^{D}\right]+K \in O\left(N^{2 D}\right)
$$

and hence our claim follows, proving the $1+\varepsilon$-subexponentiality.

More precisely, taking the subset $\alpha$ into account we wish to record here for future reference that

$$
K_{E}(N, 1+\varepsilon) \leq \sum_{k<a N^{D}, k \in \alpha} k+K
$$

We now show that $A(\alpha)$ is not exact. Recall the notation $B(\alpha)=\oplus_{m \in \alpha} M_{m}$. By Kirchberg's results (see e.g. [21, p. 286]), if $A(\alpha)$ is exact then the inclusion map $V: A(\alpha) \rightarrow B(\alpha)$ is approximable by a net of maps factoring completely positively through matrix algebras. In particular, it satisfies the following: for any $C^{*}$-algebra $C$ the mapping $V \otimes I d_{C}: A(\alpha) \otimes_{\min } C \rightarrow B(\alpha) \otimes_{\max } C$ is bounded (and is actually contractive). Let $\mathcal{U}$ be any free ultrafilter on $\alpha$. Let $M^{\mathcal{U}} \subset B\left(L_{2}\left(\tau_{\mathcal{U}}\right)\right.$ ) denote the von Neumann algebra ultraproduct of $\left\{M_{m} \mid m \in \alpha\right\}$, with each $M_{m}$ equipped with $\tau_{m}$. The Hilbert space $L_{2}\left(\tau_{\mathcal{U}}\right)$ is the one obtained by the GNS construction applied to $B(\alpha)=\oplus_{m \in \alpha} M_{m}$ equipped with the state $\tau_{\mathcal{U}}=\lim _{\mathcal{U}} \tau_{m}$. Recall that $\tau_{\mathcal{U}}$ is a faithful normal tracial state on $M^{\mathcal{U}}$ (cf. e.g. [21, p. 211]). By the weak convergence assumption, we may view $\mathcal{C}$ as embedded in $M^{\mathcal{U}}$, in such a way that $\tau_{\mathcal{U}}$ restricted to $\mathcal{C}$ coincides with $\tau$. Let $\mathcal{M}$ be the von Neumann algebra generated by $\mathcal{C}$. We have a quotient map $Q_{1}: B(\alpha) \rightarrow M^{\mathcal{U}}$ and a (completely contractive) conditional expectation $Q_{2}$ from $M^{\mathcal{U}}$ to $\mathcal{M}$. Let $q: A(\alpha) \rightarrow \mathcal{M}$ be the composition $q=Q_{2} Q_{1} V$. By the above, $q \otimes I d_{C}: A(\alpha) \otimes_{\min } C \rightarrow \mathcal{M} \otimes_{\max } C$ must be bounded (and actually contractive). However, if we take $C=\overline{\mathcal{C}}$, this implies since $c_{j}=q\left(u_{j}(\alpha)\right)$

$$
\left\|\sum_{1}^{n} c_{j} \otimes \bar{c}_{j}\right\|_{\mathcal{M} \otimes_{\max } \overline{\mathcal{C}}} \leq\left\|\sum_{1}^{n} u_{j}(\alpha) \otimes \bar{c}_{j}\right\|_{A(\alpha) \otimes_{\min } \overline{\mathcal{C}}} \leq\left\|\sum_{1}^{n} u_{j} \otimes \bar{c}_{j}\right\|_{\mathcal{A} \otimes_{\min } \overline{\mathcal{C}}}
$$


But now using the fact that left and right multiplication acting on $L_{2}\left(\tau_{\mathcal{U}}\right)$ are commuting representations on $\mathcal{M}$, we immediately find

$$
\sum_{1}^{n} \tau\left(\left|c_{j}\right|^{2}\right)=\sum_{1}^{n} \tau_{\mathcal{U}}\left(\left|c_{j}\right|^{2}\right) \leq\left\|\sum_{1}^{n} c_{j} \otimes \bar{c}_{j}\right\|_{\mathcal{M} \otimes_{\max } \overline{\mathcal{C}}}
$$

and this contradicts (7.4). This contradiction shows that $A(\alpha)$ is not exact.

Remark 7.3. More generally, let $E$ be any finite dimensional subspace spanned by a finite set of polynomials in the generators $\left\{u_{j}\right\}$. Let

$$
C_{E}(t, m)=\sup _{m^{\prime} \geq m} \sup _{k \leq t}\left\{\left\|P\left(u\left(m^{\prime}\right)\right)\right\| \mid P \in M_{k} \otimes E,\|P(c)\| \leq 1\right\} .
$$

Let $E(\alpha)$ denote the subspace of $A(\alpha)$ formed of the corresponding polynomials in the generators $\left\{u_{j}(\alpha)\right\}$ of $A(\alpha)$. The preceding proof shows more precisely that if we assume that there is a constant $C$ such that $C_{E}\left(N, a N^{D}\right) \leq C$ for all $N$ large enough then for any $c>C$ we have for any $\alpha \subset \mathbb{N}$

$$
K_{E(\alpha)}(N, c) \in O\left(\sum_{k<a N^{D}, k \in \alpha} k\right) .
$$

Remark 7.4. Let $Y^{(m)}$ denote a random $m \times m$-matrix with i.i.d. complex Gaussian entries with mean zero and $L_{2}$-norm equal to $m^{-1 / 2}$, and let $\left(Y_{j}^{(m)}\right)$ be a sequence of i.i.d. copies of $Y^{(m)}$. We will use the matrix model formed by these matrices (sometimes called the "Ginibre ensemble"), for which it is known ([29]) that we have weak convergence to a free circular family $\left\{c_{j}\right\}$. Moreover, by [9] we have also almost surely strong convergence of the random matrices to the free circular system. Actually, the inequalities from [9, 10, that we will crucially use are stated there mostly for the GUE ensemble, i.e. for self-adjoint Gaussian matrices with a semi-circular weak limit, and for self-adjoint polynomials in them with matrix coefficients. These can be defined simply by setting

$$
X_{j}^{(m)}=\sqrt{2} \Re\left(Y_{j}^{(m)}\right) .
$$

Note we also have an identity in distribution $s_{j}=\sqrt{2} \Re\left(c_{j}\right)$. We call this the self-adjoint model. However, as explained in [9], it is easy to pass from the self-adjoint case to the general one by a simple " $2 \times 2$-matrix trick". Since we prefer to work in the circular setting, with polynomials in $c_{j}, c_{j}^{*}$ (we call those $*$-polynomials) we will now indicate this trick.

When working in the self-adjoint model, of course we consider only polynomials of degree $d$ in $\left(X_{1}, \cdots, X_{n}\right)$. Fix $k$. Then the set of polynomials of degree $\leq d$ with coefficients in $M_{k}$ of the form $P\left(X_{j}^{(m)}\right)$ is included in the corresponding set of $*$-polynomials of degree $\leq d$ of the form $P\left(Y_{j}^{(m)}\right)$. Conversely, any $P\left(Y_{j}^{(m)}\right)$ can be viewed as a polynomial of degree $\leq d$ in $\left(X_{1}^{(m)}, \cdots, X_{2 n}^{(m)}\right)$. Indeed, the real and imaginary parts of $Y_{j}^{(m)}$ are independent copies of $X_{j}^{(m)}$. Thus, by this simple argument, we can replace $*$-polynomials (with coefficients in $M_{k}$ ) in $\left(Y_{j}^{(m)}\right.$ ) by polynomials (with coefficients in $M_{k}$ ) in $\left(X_{j}^{(m)}\right)$. However, there is a further restriction: The results of [9] are stated only for self-adjoint polynomials with coefficients in $M_{k}$. But then the trick (indicated in [9]) to deal with this consists in replacing a general polynomial $P \in M_{k} \otimes P_{d}$ with coefficient in $M_{k}$ by a self-adjoint one $\hat{P}$ with coefficient in $M_{2 k}$ defined by

$$
\hat{P}=\left(\begin{array}{cc}
0 & P \\
P^{*} & 0
\end{array}\right) \in M_{2 k} \otimes P_{d} .
$$


One then notes that $\|\hat{P}(s)\|=\|P(s)\|$ and similarly $\left\|\hat{P}\left(X_{j}^{(m)}\right)\right\|=\left\|P\left(X_{j}^{(m)}\right)\right\|$. Thus, for instance, by simply passing from $k$ to $2 k$ we can deduce the strong convergence for arbitrary polynomials, as expressed in (7.1) and (7.2) from the self-adjoint case.

The following Lemma is well known.

Lemma 7.5. Let $F$ be any scalar valued random variable that is in $L_{p}$ for all $p<\infty$. Fix $\theta>0$. Assume that

$$
\sup _{p \geq 1} p^{-\theta}\|F\|_{p} \leq \sigma
$$

Then

$$
\forall t>0 \quad \mathbb{P}\{|F|>t\} \leq e \exp -(e \sigma)^{-1 / \theta} t^{1 / \theta} .
$$

Proof. By Tchebyshev's inequality, for any $t>0$ we have $t^{p} \mathbb{P}\{|F|>t\} \leq\left(\sigma p^{\theta}\right)^{p}$, and hence $\mathbb{P}\{|F|>t\} \leq\left(t^{-1} \sigma p^{\theta}\right)^{p} \leq \exp -p \log \left(t /\left(\sigma p^{\theta}\right)\right)$. Assuming $t /(e \sigma) \geq 1$, we can choose $p=(t /(e \sigma))^{1 / \theta}$ and then we find $\mathbb{P}\{|F|>t\} \leq \exp -(e \sigma)^{-1 / \theta} t^{1 / \theta}$ and, a fortiori, the inequality holds. Now if $t /(e \sigma)<1$, we have $\exp -(e \sigma)^{-1 / \theta} t^{1 / \theta}>e^{-1}$ and hence $e \exp -(e \sigma)^{-1 / \theta} t^{1 / \theta}>1$ so that the inequality trivially holds.

We will use concentration of measure in the following form:

Lemma 7.6. There is a constant $c_{1}(n, d)>0$ such that for any $k$ and any $P \in M_{k} \otimes P_{d}$ with $\|P(c)\| \leq 1$, we have

$$
\forall t>0 \quad \mathbb{P}\left\{\left|\left\|P\left(Y^{(m)}\right)\right\|-\mathbb{E}\left\|P\left(Y^{(m)}\right)\right\|\right|>t\right\} \leq e \exp -\left(t^{2 / d} m^{1 / d} / c_{1}(n, d)\right) .
$$

Proof. This follows from a very general concentration inequality for Gaussian random vectors, that can be derived in various ways. We choose the following for which we refer to [18]. Consider any sufficiently smooth function (meaning a.e. differentiable) $f: \mathbb{R}^{n} \rightarrow \mathbb{R}$ and let $\mathbb{P}$ denote the canonical Gaussian measure on $\mathbb{R}^{n}$. Assuming $f \in L_{p}(\mathbb{P})$ we have

$$
\|f-\mathbb{E} f\|_{p} \leq(\pi / 2)\|D f(x) \cdot y\|_{L_{p}(\mathbb{P}(d x) \mathbb{P}(d y))} .
$$

Let $\gamma(p)$ denote the $L_{p}$-norm of a standard normal Gaussian variable (in particular $\gamma(p)=\|f\|_{p}$ for $\left.f(x)=x_{1}\right)$. Recall that $\gamma(p) \in O(\sqrt{p})$ when $p \rightarrow \infty$. Thus the last inequality implies that there is a constant $\beta$ such that

$$
\|f-\mathbb{E} f\|_{p} \leq \beta \sqrt{p}\|\| D f(x)\left\|_{2}\right\|_{L_{p}(\mathbb{P}(d x))},
$$

where $\|D f(x)\|_{2}$ denotes the Euclidean norm of the gradient of $f$ at $x$. Clearly this remains true for any $f$ on $\mathbb{C}^{n}$ (with the gradient computed on $\mathbb{R}^{2 n}$ ).

We will apply this to a function $f$ defined on $\left(\mathbb{C}^{m^{2}}\right)^{n}$. We need to first clarify the notation. We identify $\mathbb{C}^{m^{2}}$ with $M_{m}$. Let $P \in M_{k} \otimes P_{d}$. Then we define $f$ on $\left(\mathbb{C}^{m^{2}}\right)^{n}$ by

$$
f\left(w_{1}, \cdots, w_{n}\right)=\left\|g\left(w_{1}, \cdots, w_{n}\right)\right\|
$$

with

$$
g\left(w_{1}, \cdots, w_{n}\right)=P\left(m^{-1 / 2} w_{1}, \cdots, m^{-1 / 2} w_{n}, m^{-1 / 2} w_{1}^{*}, \cdots, m^{-1 / 2} w_{n}^{*}\right) .
$$

Note that for this choice of $f$ the derivative $D_{z}$ in any direction $z$ satisfies $D_{z} f \leq\left\|D_{z} g\right\|$ and hence taking the $\sup$ over $z$ in the Euclidean unit sphere, we have pointwise

$$
\|D f\|_{2} \leq \sup _{z}\left\|D_{z} g\right\|
$$


In order to majorize $\sup _{z}\left\|D_{z} g\right\|$, we first invoke Remark 7.1. Using the bound in that remark, we are reduced to majorize in the case when $P(X)=X^{J}$, a product of $\ell$ terms, with $\ell \leq d$.

Then, we claim that $D_{z} g$ is the sum of $\ell$ terms of the form $m^{-1 / 2} a z_{i} b$ satisfying, for all $z=\left(z_{i}\right)$ in the Euclidean sphere, the bound

$$
\left\|m^{-1 / 2} a z_{i} b\right\| \leq m^{-1 / 2}\|a\|\|b\| \leq m^{-1 / 2} \sup \left\{\left\|m^{-1 / 2} w_{j}\right\| \mid 1 \leq j \leq n\right\}^{\ell-1} .
$$

Indeed, if $P(X)=X^{J}=X_{j_{1}} \cdots X_{j_{\ell}}(1 \leq \ell \leq d)$, and if $g$ is associated as above (with say $\left.a_{J}=I\right)$, then $g$ is of the form $g=y_{j_{1}} \cdots y_{j_{\ell}}$ with $y_{j}=m^{-1 / 2} w_{j}, y_{n+j}=m^{-1 / 2} w_{j}^{*}$, and hence $D_{z} g=\sum_{i} y_{j_{1}} \cdots\left(D_{z} y_{j_{i}}\right) \cdots y_{j_{\ell}}$ and $D_{z} y_{j_{i}}$ is equal to $m^{-1 / 2} z_{j_{i}}$. Note $\left\|z_{i}\right\| \leq\left\|z_{i}\right\|_{2}$ and hence $\left\|a z_{i} b\right\| \leq\|a\|\|b\|$. From this the claim follows.

Recollecting all the terms, this yields a pointwise estimate at the point $w \in M_{m}^{n}$

$$
\sup _{z}\left\|D_{z} g\right\| \leq c_{3}(n, d) m^{-1 / 2} \sup \left\{\left\|m^{-1 / 2} w_{j}\right\|^{\ell-1} \mid 1 \leq j \leq n, 1 \leq \ell \leq d\right\} .
$$

Thus we obtain

$$
\|f-\mathbb{E} f\|_{p} \leq \beta \sqrt{p} c_{3}(n, d) m^{-1 / 2}\left\|\sup _{1 \leq j \leq n, \ell \leq d}\right\| Y_{j}^{(m)}\left\|^{\ell-1}\right\|_{p}
$$

and a fortiori

$$
\|f-\mathbb{E} f\|_{p} \leq \beta \sqrt{p} c_{3}(n, d) m^{-1 / 2} \sum_{1 \leq j \leq n, \ell \leq d}\|\| Y_{j}^{(m)}\left\|^{\ell-1}\right\|_{p}
$$

Now by general results on integrability of Gaussian vectors (see [13, p. 134]), we know that there is an absolute constant $c_{5}$ such that

$$
\|\| Y_{1}^{(m)}\left\|^{\ell-1}\right\|_{p}=\left\|Y_{1}^{(m)}\right\|_{L_{p(\ell-1)}^{\ell-1}\left(M_{m}\right)} \leq\left(c_{5} \sqrt{p(\ell-1)} \mathbb{E}\left\|Y_{1}^{(m)}\right\|\right)^{\ell-1}
$$

and since we know that $\mathbb{E}\left\|Y_{1}^{(m)}\right\| \rightarrow 2$ when $m \rightarrow \infty$ it follows that \|\|$Y_{1}^{(m)}\left\|^{\ell-1}\right\|_{p} \leq\left(c_{6} \sqrt{p(\ell-1)}\right)^{\ell-1} \leq$ $\left(c_{6} \sqrt{p(d-1)}\right)^{d-1}=\left(c_{6} \sqrt{(d-1)}\right)^{d-1} p^{d / 2-1 / 2}$ for some numerical constant $c_{6}>1$. Thus, by (7.7) we obtain

$$
\|f-\mathbb{E} f\|_{p} \leq c_{4}(n, d) m^{-1 / 2} p^{d / 2},
$$

and the conclusion follows from the preceding Lemma with $\theta=d / 2$ and $\sigma=c_{4}(n, d) m^{-1 / 2}$.

Remark 7.7. It will be convenient to record here an elementary consequence of Lemma 7.6, Let $F=\left\|P\left(Y^{(m)}\right)\right\|$ and let $t_{m}=\mathbb{E}\left\|P\left(Y^{(m)}\right)\right\|$, so that we know $\forall t>0 \quad \mathbb{P}\left\{F>t+t_{m}\right\} \leq \psi_{m}(t)$ with

$$
\psi_{m}(t)=e \exp -\left(t^{2 / d} m^{1 / d} / c_{1}(n, d)\right)
$$

We have

$$
\mathbb{E}\left(\left(F / 2-t_{m}\right) 1_{\left\{F / 2>t_{m}\right\}}\right)=\int_{t_{m}}^{\infty} \mathbb{P}\{F / 2>t\} d t \leq \int_{t_{m}}^{\infty} \mathbb{P}\left\{F>t+t_{m}\right\} d t \leq \int_{t_{m}}^{\infty} \psi_{m}(t) d t
$$

and hence

$$
\mathbb{E} F 1_{\left\{F / 2>t_{m}\right\}} \leq 2 t_{m} \mathbb{P}\left\{F / 2>t_{m}\right\}+2 \int_{t_{m}}^{\infty} \psi_{m}(t) d t
$$

The next result is a consequence of the results of Haagerup and Thorbjørnsen [9] and of them with Schultz [10]. Let us first recall the result from [9] that we crucially need. 
Theorem $7.8([9,10])$. Let $\chi_{d}(k, m)$ denote the best constant such that for any $P \in M_{k} \otimes P_{d}$ we have

$$
\mathbb{E}\left\|P\left(Y_{j}^{(m)}\right)\right\| \leq \chi_{d}(k, m)\|P(c)\| .
$$

Then for any $0<\delta<1 / 4$

$$
\lim _{m \rightarrow \infty} \chi_{d}\left(\left[m^{\delta}\right], m\right)=1 .
$$

Proof. Let $\chi_{d}^{\prime}(k, m)$ be defined exactly as $\chi_{d}(k, m)$ but in the self-adjoint setting, i.e. with $\left\{X_{j}^{(m)}\right\}$ in place of $\left\{Y_{j}^{(m)}\right\}$ and a free semicircular system $\left\{s_{j}\right\}$ (and $P(s)$ ) in place of $\left\{c_{j}\right\}$ (and $P(c)$ ). By Remark 7.4 it suffices to prove that $\lim _{m \rightarrow \infty} \chi_{d}^{\prime}\left(\left[m^{\delta}\right], m\right)=1$.

We will now majorize $\chi_{d}^{\prime}\left(\left[m^{\delta}\right], m\right)$. By homogeneity we may assume $\|P(s)\|=1$. Then by Remark 7.1 we also have

$$
\sum_{J}\left\|a_{J}\right\| \leq c_{2}(n, d)
$$

Fix $\varepsilon>0$ and $t>1+\varepsilon$. Consider a function $\varphi \in C_{c}^{\infty}(\mathbb{R}, \mathbb{R})$ with values in $[0,1]$ such that $\varphi=0$ on $[-1,1]$ and $\varphi(x)=1$ for all $x$ such that $1+\varepsilon<|x|<t$ and $\varphi(x)=0$ for $|x|>2 t$. Let $P^{(m)}=P\left(X_{j}^{(m)}\right)$ and $P^{(\infty)}=P\left(s_{j}\right)$. By Remark 7.4 we can reduce our estimate to the case of a self-adjoint polynomial in $\left(X_{j}^{(m)}\right)$. Then by [10] (and by very carefully tracking the dependence of the various constants in [10]) we have for $m \geq c_{13}(n, d)$

$$
\left.\mathbb{E}\left\{\left(\tau_{k} \otimes \tau_{m}\right) \varphi\left(P^{(m)}\right)\right\}=\left(\tau_{k} \otimes \tau\right) \varphi\left(P^{(\infty)}\right)\right)+R_{m}(\varphi)
$$

where

$$
\left|R_{m}(\varphi)\right| \leq k^{3} m^{-2} c_{9}(n, d) c_{\varepsilon} t^{3}
$$

where $c_{\varepsilon}$ depends only on $\varepsilon$. Note $\varphi\left(P^{(\infty)}\right)=0$. Therefore

$$
\mathbb{E}\left\{\left(\tau_{k} \otimes \tau_{m}\right) \varphi\left(P^{(m)}\right)\right\} \leq k^{3} m^{-2} c_{9}(n, d) c_{\varepsilon} t^{3} .
$$

Since $\left\|P^{m}\right\| \in(1+\varepsilon, t) \Rightarrow\left(\tau_{k} \otimes \tau_{m}\right) \varphi\left(P^{m}\right) \geq 1 /(k m)$ by Tchebyshev's inequality we find

$$
\mathbb{P}\left\{\left\|P^{(m)}\right\| \in(1+\varepsilon, t)\right\} \leq(k m) k^{3} m^{-2} c_{9}(n, d) c_{\varepsilon} t^{3}=k^{4} m^{-1} c_{9}(n, d) c_{\varepsilon} t^{3} .
$$

Thus we obtain

$$
\mathbb{E}\left\|P^{(m)}\right\| \leq 1+\varepsilon+k^{4} m^{-1} c_{9}(n, d) c_{\varepsilon} t^{4}+\mathbb{E}\left(\left\|P^{(m)}\right\| 1_{\left\{\left\|P^{(m)}\right\|>t\right\}}\right) .
$$

We will now invoke (7.8): choosing $t=2 t_{m}=2 \mathbb{E}\left\|P^{(m)}\right\|$ we find

$$
\mathbb{E}\left\|P^{(m)}\right\| \leq 1+\varepsilon+k^{4} m^{-1} c_{9}(n, d) c_{\varepsilon} t_{m}^{4}+2 t_{m} \psi_{m}\left(t_{m}\right)+2 \int_{t_{m}}^{\infty} \psi_{m}(t) .
$$

Now by (7.9) and by Hölder we have

$$
t_{m} \leq c_{2}(n, d) \sup _{J} \mathbb{E}\left\|X^{(m)^{J}}\right\| \leq c_{2}(n, d) \sup _{|J| \leq d} \mathbb{E}\left(\left\|X_{1}^{(m)}\right\|^{|J|}\right)
$$

but by a well known result essentially due to Geman [6] (cf. e.g. [28, Lemma 6.4]), for any $d$ we have

$$
c_{9}(d)=\sup _{m} \mathbb{E}\left(\left\|X_{1}^{(m)}\right\|^{d}\right)<\infty
$$


Therefore we have $t_{m} \leq c_{2}^{\prime}(n, d)$. We may assume $t_{m}>1$ (otherwise there is nothing to prove) and hence we have proved

$$
\mathbb{E}\left\|P^{(m)}\right\| \leq 1+\varepsilon+k^{4} m^{-1} c_{9}^{\prime}(n, d) c_{\varepsilon}+2 c_{2}^{\prime}(n, d) \psi_{m}(1)+2 \int_{1}^{\infty} \psi_{m}(t) d t .
$$

Thus for any $\varepsilon>0$ we conclude

$$
\chi_{d}^{\prime}(k, m) \leq 1+\varepsilon+k^{4} m^{-1} c_{9}^{\prime}(n, d) c_{\varepsilon}+2 c_{2}^{\prime}(n, d) \psi_{m}(1)+2 \int_{1}^{\infty} \psi_{m}(t) d t .
$$

From this estimate it follows clearly that for any $0<\delta<1 / 4$

$$
\limsup _{m \rightarrow \infty} \chi_{d}^{\prime}\left(\left[m^{\delta}\right], m\right) \leq 1+\varepsilon
$$

Lemma 7.9. Fix integers $d, k, m$. Let $\chi_{d}(k, m)$ denote the best constant appearing in Theorem 7.8 . Then for any $\varepsilon>0$ there are positive constants $c_{7}(n, d, \varepsilon)$ and $c_{8}(n, d, \varepsilon)$ such that if $k$ is the largest integer such that $m \geq c_{7}(n, d, \varepsilon) k^{2 d}$ the set

$$
\Omega_{d, \varepsilon}(m)=\left\{\forall P \in M_{k} \otimes P_{d} \quad\left\|P\left(Y^{(m)}(\omega)\right)\right\| \leq(1+\varepsilon)\left(\chi_{d}(k, m)+\varepsilon\right)\|P(c)\|\right\}
$$

satisfies

$$
\mathbb{P}\left(\Omega_{d, \varepsilon}(m)^{c}\right) \leq e \exp \left(-m^{1 / d} / c_{8}(n, d, \varepsilon)\right) .
$$

Proof. For any $P \in M_{k} \otimes P_{d}$ with $\|P(c)\| \leq 1$, we have by Lemma 7.6 for any $t>0$

$$
\mathbb{P}\left\{\left\|P\left(Y^{(m)}\right)\right\|>t+\chi_{d}(k, m)\right\} \leq e \exp -\left(t^{2 / d} m^{1 / d} / c_{1}(n, d)\right) .
$$

Let $\mathcal{N}$ be a $\delta$-net in the unit ball of the space $P_{d}$ equipped with the norm $P \mapsto\|P(c)\|$. Since $\operatorname{dim}\left(M_{k} \otimes P_{d}\right)=c_{6}(n, d) k^{2}$ for some $c_{6}(n, d)$, it is known that we can find such a net with

$$
|\mathcal{N}| \leq(1+2 / \delta)^{c_{6}(n, d) k^{2}} .
$$

Let $\Omega_{1}=\left\{\forall a \in \mathcal{N},\left\|P\left(Y^{(m)}\right)\right\|>t+\chi_{d}(k, m)\right\}$. Clearly

$$
\mathbb{P}\left(\Omega_{1}\right) \leq|\mathcal{N}| e \exp -\left(t^{2 / d} m^{1 / d} / c_{1}(n, d)\right) \leq e \exp \left(2 c_{6}(n, d) \delta^{-1} k^{2}-t^{2 / d} m^{1 / d} / c_{1}(n, d)\right) .
$$

Thus if we choose $m$ so that (roughly ) $t^{2 / d} m^{1 / d} / c_{1}(n, d)=4 c_{6}(n, d) \delta^{-1} k^{2}$ we find an estimate of the form

$$
\mathbb{P}\left(\Omega_{1}\right) \leq e \exp \left(-t^{2 / d} m^{1 / d} / 2 c_{1}(n, d)\right) .
$$

Note that on the complement of $\Omega_{1}$ we have

$$
\forall P \in \mathcal{N} \quad\left\|P\left(Y^{(m)}\right)\right\| \leq t+\chi_{d}(k, m) .
$$

By a well known result (see e.g. [19, p. 49-50]) we can pass from the set $\mathcal{N}$ to the whole unit ball at the cost of a factor close to 1 , namely we have on the complement of $\Omega_{1}$

$$
\forall P \in M_{k} \otimes P_{d} \quad\left\|P\left(Y^{(m)}\right)\right\| \leq(1-\delta)^{-1}\left(t+\chi_{d}(k, m)\right)\|P(c)\| .
$$


Thus if we set $t=\varepsilon$ and $\delta \approx \varepsilon / 2$, we obtain that if $m \geq c_{7}(n, d, \varepsilon) k^{2 d}$ we have a set $\Omega_{1}^{\prime}=\Omega_{1}^{c}$ with

$$
\mathbb{P}\left(\Omega_{1}^{\prime c}\right) \leq e \exp \left(-\varepsilon^{2 / d} m^{1 / d} / 2 c_{1}(n, d)\right),
$$

such that for any $\omega \in \Omega_{1}^{\prime}$ we have

$$
\forall P \in M_{k} \otimes P_{d} \quad\left\|P\left(Y^{(m)}(\omega)\right)\right\| \leq(1+\varepsilon)\left(\chi_{d}(k, m)+\varepsilon\right)\|P(c)\| .
$$

Theorem 7.10. For any infinite subset $\alpha \subset \mathbb{N}$ and each $j$ let $u_{j}(\alpha)(\omega)$ denote the block direct sum defined by

$$
u_{j}(\alpha)(\omega)=\oplus_{m \in \alpha} Y_{j}^{(m)}(\omega) \in \oplus_{m \in \alpha} M_{m},
$$

and let $u_{j}(\omega)=u_{j}(\mathbb{N})(\omega)$. Let $A(\alpha)(\omega)$ denote the $C^{*}$-algebra generated by the infinite sequence $\left\{u_{j}(\alpha)(\omega) \mid j=1,2, \cdots\right\}$. Then, for almost every $\omega$, the $C^{*}$-algebras $A(\alpha)(\omega)$ are all subexponential with constant 1 but are not exact.

Moreover, these results remain valid in the self-adjoint setting, if we replace $u_{j}(\alpha)(\omega)$ by

$$
\hat{u}_{j}(\alpha)(\omega)=\oplus_{m \in \alpha} X_{j}^{(m)}(\omega) \in \oplus_{m \in \alpha} M_{m} .
$$

Proof. By Lemma 7.9 for any degree $d$ and $\varepsilon>0$ we have

$$
\sum_{m} \mathbb{P}\left(\Omega_{d, \varepsilon}(m)^{c}\right)<\infty .
$$

Therefore the set $V_{d, \varepsilon}=\liminf _{m \rightarrow \infty} \Omega_{d, \varepsilon}(m)$ has probability 1. Furthermore (since we may use a sequence of $\varepsilon$ 's tending to zero) we have

$$
\mathbb{P}\left(\cap_{d, \varepsilon} V_{d, \varepsilon}\right)=1 .
$$

Now if we choose $\omega$ in $\cap_{d \geq 1, \varepsilon>0} V_{d, \varepsilon}$, by Theorem 7.8 , the operators $u_{j}(\omega)$ satisfy the assumptions of Theorem 7.2, and hence $A(\alpha)(\omega)$ is 1-subexponential for any $\alpha$.

Recall that, by concentration (see Remark 7.11 below)

$$
\sup _{j \geq 1} \mathbb{E}\left(\left\|u_{j}\right\|^{2}\right)=\sup _{j \geq 1} \mathbb{E}\left(\sup _{m}\left\|u_{j}(m)\right\|^{2}\right)<\infty .
$$

Therefore, by Fatou's lemma

$$
\mathbb{E} \liminf _{n \rightarrow \infty} n^{-1} \sum_{1}^{n}\left\|u_{j}\right\|^{2} \leq \liminf _{n \rightarrow \infty} \mathbb{E} n^{-1} \sum_{1}^{n}\left\|u_{j}\right\|^{2}<\infty
$$

and hence there is a measurable set $\Omega_{0} \subset \Omega$ with $\mathbb{P}\left(\Omega_{0}\right)=1$ such that

$$
\forall \omega \in \Omega_{0} \quad \liminf _{n \rightarrow \infty} n^{-1} \sum_{1}^{n}\left\|u_{j}(\omega)\right\|^{2}<\infty .
$$

Therefore if we choose $\omega$ in the intersection of $\cap_{d, \varepsilon} V_{d, \varepsilon} \cap \Omega_{0}$ (which has probability 1) we find almost surely by (1.4)

$$
\left\|\sum_{1}^{n} u_{j}(\omega) \otimes \bar{c}_{j}\right\|_{\mathcal{A} \otimes_{\min } \overline{\mathcal{C}}} \leq 2 \max \left\{\left\|\sum u_{j} u_{j}^{*}\right\|^{1 / 2},\left\|\sum u_{j}^{*} u_{j}\right\|^{1 / 2}\right\} \leq 2\left(\sum_{1}^{n}\left\|u_{j}(\omega)\right\|^{2}\right)^{1 / 2} \in O(\sqrt{n})
$$

so that (7.4) is satisfied when $n$ is large enough and hence $A(\alpha)(\omega)$ is not exact.

Lastly, since $\left\{\hat{u}_{j}(\alpha)(\omega) \mid j \in \alpha\right\}$ has the same distribution as $\left\{\sqrt{2} \Re u_{j}(\alpha)(\omega) \mid j \in \alpha\right\}$ the random $C^{*}$-algebra they generate has "the same distribution" as $A(\alpha)(\omega)$, whence the last assertion. 
Remark 7.11. In the preceding proof, we use the fact that $\sup _{j>1} \mathbb{E}\left(\left\|u_{j}\right\|^{2}\right)<\infty$. This is immediate if we assume that the vector valued random variables $\left\{u_{j} \mid j \geq 1\right\}$ are (stochastically) independent, since then they have automatically the same distribution so this reduces to $\mathbb{E}\left(\left\|u_{1}\right\|^{2}\right)<\infty$. However, we claim this remains valid assuming merely, as we do, that for each $m$ the sequence $\left\{u_{j}(m) \mid j \geq 1\right\}$ is independent. This follows rather easily from the common concentration of the variables $\left\{u_{j} \mid\right.$ $j \geq 1$ \}. Indeed, by a well known (rather soft) bound we have

$$
\Delta=\sup _{j, m \geq 1} \mathbb{E}\left\|u_{j}(m)\right\|<\infty .
$$

Then by (2.3) we have for any $j \geq 1$

$$
\forall t>0 \quad \mathbb{P}\left\{\left\|u_{j}(m)\right\|>t+\Delta\right\} \leq 2 \exp -t^{2} m
$$

and hence for all $t>0$

$$
\mathbb{P}\left\{\left\|u_{j}\right\|>t+\Delta\right\} \leq \sum_{m} \mathbb{P}\left\{\left\|u_{j}(m)\right\|>t+\Delta\right\} \leq 2 \sum_{m} \exp -t^{2} m \leq 2\left(\exp -t^{2}\right)\left(1-\exp -t^{2}\right)^{-1},
$$

from which our claim is immediate.

Remark 7.12. It seems clear that our results remain valid if we replace $\left(Y_{j}^{(m)}\right)$ by an i.i.d. sequence $\left(V_{j}^{(m)}\right)$ of uniformly distributed $m \times m$ unitary matrices, and we replace $u_{j}$ by

$$
v_{j}(\alpha)(\omega)=\oplus_{m \in \alpha} V_{j}^{(m)}(\omega) \subset \oplus_{m \in \alpha} M_{m} .
$$

Note that Collins and Male [3] proved that strong convergence holds in this case. But, at the time of this writing, except for a partial result in Theorem 8.11 below, we have not been able to prove that $\left(V_{j}^{(m)}\right)$ satisfies the same estimates (e.g. as in Theorem 7.8$)$ as $\left(Y_{j}^{(m)}\right)$.

However, as this paper was being completed, Mikael de la Salle kindly communicated to me the proof of the following result.

Theorem 7.13 (de la Salle). Let $\alpha \subset \mathbb{N}$. Let $A^{U}(\alpha)(\omega)$ be the $C^{*}$-algebra generated by the unitary operators $\left\{v_{j}(\alpha)(\omega) \mid 1 \leq j \leq n\right\}$. Then $A^{U}(\alpha)(\omega)$ is subexponential with constant 1 .

Sketch of proof. Let $c$ be a circular variable, so that $s=\sqrt{2} \Re(c)$ is semi-circular. Let $f: \mathbb{R} \rightarrow \mathbb{T}$ be a continuous function such that $f(s)$ is uniformly distributed over $\mathbb{T}$. By [3] we may assume $\left(Y_{j}^{(m)}\right)$ and $\left(V_{j}^{(m)}\right)$ defined on a suitably enlarged probability space so that $\left\|f\left(\sqrt{2} Y_{j}^{(m)}\right)-V_{j}^{(m)}\right\| \rightarrow 0$ almost surely when $m \rightarrow \infty$. Then by [3] the family $\left(f\left(\sqrt{2} Y_{j}^{(m)}\right)\right)$ converges strongly to $\left(f\left(s_{j}\right)\right)$ and the latter is a family of free Haar unitaries. Let $C_{d}^{f}\left(N, a N^{D}\right)$ be the analogue of $C_{d}\left(N, a N^{D}\right)$ but computed with $Y_{j}^{(m)}$ replaced by $f\left(\sqrt{2} Y_{j}^{(m)}\right)$ and by $\left(c_{j}\right)$ replaced by $\left(f\left(s_{j}\right)\right)$. By the proof of Theorem 7.2 it suffices to show the following claim: For any $d$ and $\varepsilon>0$ there is $a, D$ such that $\limsup _{N \rightarrow \infty} C_{d}^{f}\left(N, a N^{D}\right) \leq 1+\varepsilon$. Assume for a moment (although this is clearly wrong) that $f$ is a polynomial of degree $q$. Then since a polynomial of degree $d$ in $\left(f\left(s_{j}\right)\right)$ is also one of degree $\leq q d$ in $\left(c_{j}\right)$, we have $C_{q d}^{f}\left(N, a N^{D}\right) \leq C_{d}\left(N, a N^{D}\right)$, and the claim follows. To prove the claim when $f$ is not a polynomial, one approximates $f$ uniformly on (say) the interval $[-4,4]$ (which contains $[-2,2]$ in its interior) by a polynomial $P$. Since $\left\|\sqrt{2} Y_{j}^{(m)}\right\| \rightarrow 2$, when $m$ is large, we can approximate $f\left(\sqrt{2} Y_{j}^{(m)}\right)$ by $P\left(\sqrt{2} Y_{j}^{(m)}\right)$.

Remark 7.14. By a unitary variant of the argument for non-exactness in Theorem 7.10, it is easy to see that, whenever $\alpha$ is infinite and $n>2, A^{U}(\alpha)(\omega)$ is almost surely not exact (indeed note that if $\left(z_{j}\right)$ are free Haar unitaries we have $\left\|\sum_{1}^{n} v_{j}(\alpha)(\omega) \otimes \bar{z}_{j}\right\|=2 \sqrt{n-1}>n$ for any $\left.\omega\right)$. 


\section{More examples of non-exact subexponential operator spaces}

In this $\oint$ we modify the preceding example to produce $n$-dimensional operator spaces $E$ with large exactness constant such that their associated sequence $K_{E}(N, C)$ grows as slowly as possible. We will obtain a growth of order $O\left(N^{2}\right)$ when $N \rightarrow \infty$. So far this is the slowest growth we could produce among spaces with large exactness constant.

By Theorem 7.8 and Lemma 0.1, we can apply Remark 7.3 to the linear span of the generators with $a=1$ and $D$ equal to any number $>4$. This shows that if we consider $E=E_{1}(\alpha)$ then for any $\varepsilon, \delta>0$ and any $\alpha$ and we have $K_{E}(N, 1+\varepsilon) \in O\left(N^{5+\delta}\right)$ when $N \rightarrow \infty$. However, when $\alpha$ is a lacunary sequence (or when $1+\varepsilon$ is replaced by $2+\varepsilon$ ), we will show that this estimate can be improved.

Lemma 8.1. Let $E(\omega) \subset A(\omega)$ be the linear span of $\left\{u_{1}(\omega), \cdots, u_{n}(\omega)\right\}$ in $A(\omega)$. With the notation in Remark 7.3, for any $\varepsilon>0$ there is a constant $c_{\varepsilon} \geq 1$ (depending only on $\varepsilon$ ) and a (measurable) subset $\Omega_{2} \subset \Omega$ with $P\left(\Omega_{2}\right)=1$ such that for all $\omega \in \Omega_{2}$ we have for all $N$ large enough (i.e. $\left.\forall N \geq N_{0}(\varepsilon, \omega)\right)$

$$
C_{E(\omega)}\left(N, c_{\varepsilon} n N^{2}\right) \leq 2+\varepsilon .
$$

Proof. To simplify the notation, let $k_{m}=\left[\left(m /\left(c_{\varepsilon} n\right)\right)^{1 / 2}\right]$. By Lemma 0.1 for each $m$ there is $\Omega(m) \subset \Omega$ with $\mathbb{P}(\Omega(m))>1-3^{-2 n k_{m}{ }^{2}}$ such that for any $\omega \in \Omega(m)$ and any $k \leq k_{m}$ (equivalently $\left.c_{\varepsilon} n k^{2} \leq m\right)$ we have

$$
\forall\left(a_{j}\right) \in M_{k}^{n} \quad\left\|\sum a_{j} \otimes u_{j}^{(m)}(\omega)\right\| \leq(2+\varepsilon) \max \left\{\left\|\left(\sum a_{j}^{*} a_{j}\right)^{1 / 2}\right\|,\left\|\left(\sum a_{j} a_{j}^{*}\right)^{1 / 2}\right\|\right\} .
$$

and a fortiori by (1.4)

$$
\forall P \in M_{k} \otimes E \quad \| P\left(\left\{u_{i}^{(m)}\right\}\|\leq(2+\varepsilon)\| P(c) \| .\right.
$$

Since $\sum_{m} 3^{-2 n k_{m}{ }^{2}}<\infty$, it follows that for almost all $\omega \in \Omega$ (8.1) must hold for all $m$ large enough, and a fortiori also (8.2). This implies that $C_{E(\omega)}\left(N,\left[c_{\varepsilon} n N^{2}\right]\right) \leq 2+\varepsilon$ for all $N$ large enough.

Remark 8.2. It should be possible to use Collins and Male's results [3] to replace Gaussian random matrices by unitary ones (uniformly distributed according to Haar measure), but we could not check this. For this the Gromov-Lévy isoperimetric inequality (see [7, $\S 1.2$ and $\S 3.4]$ ) should be used on $U(N)^{n}$ instead of the Gaussian concentration of measure.

Fix $0<\varepsilon<1$. We may replace $c_{\varepsilon}$ by a larger number, so we will assume for simplicity that $c_{\varepsilon}$ is an integer, and we denote $a=c_{\varepsilon} n$. Let us choose $N(m)$ inductively such that $N(0)=1$ and for any $m>0$

$$
N(m+1)=c_{\varepsilon} n N(m)^{2}=a N(m)^{2} .
$$

Thus

$$
\forall m \geq 0 \quad N(m)=a^{2^{m}-1}
$$

Lemma 8.3. Let $\alpha_{2}=\{N(m) \mid m \geq 0\}$. Then for all $\omega \in \Omega_{2}$, the space $E\left(\alpha_{2}\right)(\omega)$ satisfies $K_{E\left(\alpha_{2}\right)(\omega)}(N, 2+\varepsilon) \in O\left(N^{2}\right)$ when $N \rightarrow \infty$. Moreover, this also holds for $E\left(\alpha^{\prime}\right)(\omega)$ for any subset $\alpha^{\prime} \subset \alpha_{2}$. 
Proof. By Remark 7.3 and Lemma 8.1 we have for all $N$ large enough

$$
K_{E(\alpha)(\omega)}(N, 2+\varepsilon) \leq \sum_{m \geq 0, N(m)<a N^{2}} N(m)
$$

and $N(m)<a N^{2}$ iff $N(m-1)<N$, so that

$$
\sum_{m \geq 0, N(m)<a N^{2}} N(m)=\sum_{m \geq 0, N(m-1)<N} a^{2^{m}-1}=N(0)+N(1)+\cdots+N(q)
$$

where $q$ is so that $N(q-1)<N \leq N(q)$. Now there is clearly a constant $\gamma$ such that $N(0)+N(1)+\cdots+N(q) \leq \gamma a^{2^{q}-1} \leq \gamma a N(q-1)^{2}<\gamma a N^{2}$, so that we find

$$
K_{E(\alpha)(\omega)}(N, 2+\varepsilon)<\gamma a N^{2} .
$$

By (0.5) and (1.4), we know there is a (measurable) subset $\Omega_{3} \subset \Omega$ with $P\left(\Omega_{3}\right)=1$ such that for any $\omega \in \Omega_{3}$, any $k$ and any $a_{j} \in M_{k}$ we have

$$
\max \left\{\left\|\left(\sum a_{j}^{*} a_{j}\right)^{1 / 2}\right\|,\left\|\left(\sum a_{j} a_{j}^{*}\right)^{1 / 2}\right\|\right\} \leq \liminf _{N \rightarrow \infty}\left\|\sum_{1}^{n} Y_{j}^{(N)} \otimes a_{j}\right\| .
$$

Indeed, we can easily reduce this to the countable set of all $a_{j}$ 's with entries in (say) $\mathbb{Q}+i \mathbb{Q}$. A fortiori, for any $\omega \in \Omega_{3}$ any $k$, any $a_{j} \in M_{k}$ and any infinite subset $\alpha \subset \mathbb{N}$ we have

$$
\max \left\{\left\|\left(\sum a_{j}^{*} a_{j}\right)^{1 / 2}\right\|,\left\|\left(\sum a_{j} a_{j}^{*}\right)^{1 / 2}\right\|\right\} \leq\left\|\sum a_{j} \otimes u_{j}(\alpha)(\omega)\right\| .
$$

Thus the mapping $v: E(\alpha)(\omega) \rightarrow R_{n}$ (resp. $w: E(\alpha)(\omega) \rightarrow C_{n}$ ) defined by $v\left(u_{j}(\alpha)(\omega)\right)=e_{1 j}$ $\left(\right.$ resp. $\left.w\left(u_{j}(\alpha)(\omega)\right)=e_{j 1}\right)$ satisfies

$$
\|v\|_{c b} \leq 1 \quad\left(\text { resp. }\|w\|_{c b} \leq 1\right) .
$$

Let $\Omega^{\prime \prime} \subset \Omega$ be the set of all $\omega$ 's such that

$$
\limsup _{m \rightarrow \infty}\left\|\left(Y_{j}^{(N(m))}(\omega)\right)\right\|_{R C} \leq 2 \sqrt{n} .
$$

Clearly $\mathbb{P}\left(\Omega^{\prime \prime}\right)=1$ (Indeed this follows a fortiori from Lemma 0.1).

Let $\Omega^{\prime \prime \prime} \subset \Omega$ be the set of all $\omega$ 's such that for any matrix $a \in M_{n}$ we have

$$
|\operatorname{tr}(a)| \leq \liminf _{m \rightarrow \infty}\left|\sum_{i j} a_{i j} N(m)^{-1} \operatorname{tr}\left(Y_{i}^{(N(m))} Y_{j}^{(N(m))^{*}}\right)\right| .
$$

The convergence in moments (to a circular family) of $Y_{j}^{(N(m))}$ when $m \rightarrow \infty$ ensures that this event has full probability for any fixed $a$, but again a density argument (in $M_{n}$ ) ensures that $\mathbb{P}\left(\Omega^{\prime \prime \prime}\right)=1$.

For convenience we denote

$$
\Delta_{j}=e_{1 j} \oplus e_{j 1} \in R_{n} \oplus C_{n} .
$$

We now choose $\omega$ in the set $\Omega_{2} \cap \Omega_{3} \cap \Omega^{\prime \prime} \cap \Omega^{\prime \prime \prime}$ which occurs with full probability and we set

$$
x_{j}(m)=Y_{j}^{(N(m))}(\omega) \in M_{N(m)} .
$$


By the choice of $\omega$ we know that for some $m_{0}>1$ we have for any $m \geq m_{0}-1$ and any $\left(y_{j}\right) \in M_{N(m)}^{n}$

$$
\|y\|_{R C} \leq \sup _{m^{\prime}>m}\left\|\sum x_{j}\left(m^{\prime}\right) \otimes y_{j}\right\| \leq(2+\varepsilon)\|y\|_{R C}=(2+\varepsilon)\left\|\sum \Delta_{j} \otimes y_{j}\right\|
$$

so that

$$
\sup _{1 \leq m^{\prime} \leq m}\left\|\sum x_{j}\left(m^{\prime}\right) \otimes y_{j}\right\| \leq\left\|\sum x_{j} \otimes y_{j}\right\| \leq \max \left\{\sup _{1 \leq m^{\prime} \leq m}\left\|\sum x_{j}\left(m^{\prime}\right) \otimes y_{j}\right\|,(2+\varepsilon)\left\|\sum \Delta_{j} \otimes y_{j}\right\|\right\}
$$

For convenience we may as well assume $m_{0}$ large enough so that $N\left(m_{0}-1\right) \geq 2 n$. Then (8.5) implies that for any $\left(y_{j}\right) \in\left(R_{n} \oplus C_{n}\right)^{n}$

$$
\sup _{m^{\prime} \geq m_{0}}\left\|\sum x_{j}\left(m^{\prime}\right) \otimes y_{j}\right\| \leq(2+\varepsilon)\left\|\sum \Delta_{j} \otimes y_{j}\right\| .
$$

For any subset $\alpha \subset \mathbb{N}$, we will denote

$$
x_{j}(\alpha)=\oplus_{m \in \alpha} x_{j}(m) \quad \text { and } \quad E_{\alpha}=\operatorname{span}\left\{x_{1}(\alpha), \cdots, x_{n}(\alpha)\right\} .
$$

The following was used in [12] for diagonal matrices, the general case was observed in [16].

Lemma 8.4. With the above notation, let $\alpha, \beta \subset\left[m_{0}, \infty\right)$ be infinite such that $\alpha \backslash \beta$ is infinite. For any $\left[a_{i j}\right] \in M_{n}$ let $T: E_{\beta} \rightarrow E_{\alpha}$ be defined by $T\left(x_{j}(\beta)\right)=\sum_{i} a_{i j} x_{i}(\alpha)$. Then we have

$$
(2+\varepsilon)^{-2}\left(\sum\left|a_{i j}\right|^{2}\right)^{1 / 2} \leq\|T\|_{C B\left(E_{\beta}, E_{\alpha}\right)} \leq(2+\varepsilon)\left(\sum\left|a_{i j}\right|^{2}\right)^{1 / 2} .
$$

Proof. Since $\omega \in \Omega_{3}$, (8.4) ensures a fortiori that we have a completely contractive natural map $E_{\beta} \rightarrow R_{n}$ (and also $E_{\beta} \rightarrow C_{n}$ ) for any infinite $\beta$. Therefore $\|T\|_{C B\left(E_{\beta}, E_{\alpha}\right)} \leq\|T\|_{C B\left(R_{n}, E_{\alpha}\right)}=$ $\left\|\sum a_{j i} e_{i 1} \otimes u_{j}(\alpha)(\omega)\right\|$ and by (8.7)

$$
\left\|\sum a_{j i} e_{i 1} \otimes u_{j}(\alpha)(\omega)\right\| \leq(2+\varepsilon)\left(\sum\left|a_{i j}\right|^{2}\right)^{1 / 2} .
$$

Therefore $\|T\|_{C B\left(E_{\beta}, E_{\alpha}\right)} \leq(2+\varepsilon)\left(\sum\left|a_{i j}\right|^{2}\right)^{1 / 2}$.

To prove the converse, we will choose $m_{0}^{\prime} \geq m_{0}$ arbitrarily large in $\alpha \backslash \beta$. Then (8.5) and (8.7) show that for any scalar matrix $\left[b_{j k}\right]$

$$
\left\|\sum b_{j k} x_{j}\left(\beta \cap\left(m_{0}^{\prime}, \infty\right)\right) \otimes \overline{x_{k}\left(m_{0}^{\prime}\right)}\right\| \leq(2+\varepsilon)\left\|\sum b_{j k} \Delta_{j} \otimes \overline{x_{k}\left(m_{0}^{\prime}\right)}\right\| \leq(2+\varepsilon)^{2}\left\|\sum b_{j k} \Delta_{j} \otimes \overline{\Delta_{k}}\right\|,
$$

and also since $m_{0}^{\prime} \notin \beta$ again by (8.5) and (8.7)

$$
\begin{gathered}
\left\|\sum b_{j k} x_{j}\left(\beta \cap\left[m_{0}, m_{0}^{\prime}\right]\right) \otimes \overline{x_{k}\left(m_{0}^{\prime}\right)}\right\| \leq(2+\varepsilon)\left\|\sum b_{j k} x_{j}\left(\beta \cap\left[m_{0}, m_{0}^{\prime}\right]\right) \otimes \overline{\Delta_{k}}\right\| \\
\leq(2+\varepsilon)^{2}\left\|\sum b_{j k} \Delta_{j} \otimes \overline{\Delta_{k}}\right\| .
\end{gathered}
$$

Recollecting the two preceding estimates, we find

$$
\left\|\sum b_{j k} x_{j}(\beta) \otimes \overline{x_{k}\left(m_{0}^{\prime}\right)}\right\| \leq(2+\varepsilon)^{2}\left\|\sum b_{j k} \Delta_{j} \otimes \overline{\Delta_{k}}\right\|=(2+\varepsilon)^{2}\left(\sum_{j k}\left|b_{j k}\right|^{2}\right)^{1 / 2}
$$

Thus we have

$$
\left\|\sum_{i j k} b_{j k} a_{i j} x_{i}(\alpha) \otimes \overline{x_{k}\left(m_{0}^{\prime}\right)}\right\|=\left\|\sum b_{j k} T\left(x_{j}(\beta)\right) \otimes \overline{x_{k}\left(m_{0}^{\prime}\right)}\right\| \leq\|T\|_{c b}(2+\varepsilon)^{2}\left(\sum_{j k}\left|b_{j k}\right|^{2}\right)^{1 / 2} .
$$


Since $m_{0}^{\prime} \in \alpha$, a fortiori

$$
\left\|\sum_{i j k} b_{j k} a_{i j} x_{i}\left(m_{0}^{\prime}\right) \otimes \overline{x_{k}\left(m_{0}^{\prime}\right)}\right\| \leq\|T\|_{c b}(2+\varepsilon)^{2}\left(\sum_{j k}\left|b_{j k}\right|^{2}\right)^{1 / 2} .
$$

But now, since $\omega \in \Omega^{\prime \prime \prime}$, for any $\delta>0$, when $m_{0}^{\prime}$ is chosen large enough, we have

$$
\left|\sum_{i j} a_{i j} b_{j i}\right| \leq\left\|\sum_{i j k} b_{j k} a_{i j} x_{i}\left(m_{0}^{\prime}\right) \otimes \overline{x_{k}\left(m_{0}^{\prime}\right)}\right\|+\delta
$$

and hence choosing simply $b_{i j}=\bar{a}_{j i}$ (and letting $\delta \rightarrow 0$ ) we conclude

$$
\sum_{i j}\left|a_{i j}\right|^{2} \leq\|T\|_{c b}(2+\varepsilon)^{2}\left(\sum_{i j}\left|a_{i j}\right|^{2}\right)^{1 / 2}
$$

and the announced result follows after division.

Lemma 8.5. Fix $m \geq m_{0}-1$. Assume that $\alpha, \beta$ are infinite subsets such that $\alpha \cap[0, m]=\beta \cap[0, m]$. Then the mapping $T: E_{\beta} \rightarrow E_{\alpha}$ (induced by the identity of $\mathbb{C}^{n}$ ) is such that

$$
\forall k \leq N(m) \quad\left\|T_{k}: M_{k}\left(E_{\beta}\right) \rightarrow M_{k}\left(E_{\alpha}\right)\right\| \leq 2+\varepsilon
$$

Proof. For all $y \in M_{k}^{n}$ we have

$$
\left\|\sum x_{j}(\alpha \cap[0, m]) \otimes y_{j}\right\|=\left\|\sum x_{j}(\beta \cap[0, m]) \otimes y_{j}\right\| \leq\left\|\sum x_{j}(\beta) \otimes y_{j}\right\| .
$$

But also by (8.5) since $k \leq N(m)$ and $\omega \in \Omega_{3}$

$$
\left\|\sum x_{j}(\alpha \cap[m+1, \infty)) \otimes y_{j}\right\| \leq(2+\varepsilon)\|y\|_{R C} \leq(2+\varepsilon)\left\|\sum x_{j}(\beta) \otimes y_{j}\right\| .
$$

Therefore $\left\|T_{k}\right\| \leq 2+\varepsilon$.

The method of the paper [12] as presented in [21, Th. 21.13, p. 343] shows that there is a continuous subcollection in the family $\left\{E_{\alpha}|\alpha \subset \mathbb{N},| \alpha \mid=\infty\right\}$ formed of spaces $E$ 's such that $\operatorname{ex}(E) \geq \sqrt{n} /(2+\varepsilon)^{3}$. But actually we can make this slightly more precise:

Theorem 8.6. For any infinite subset $\alpha \subset\left[m_{0}, \infty\right)$ we have

$$
\operatorname{ex}\left(E_{\alpha}\right) \geq \sqrt{n} /(2+\varepsilon)^{3} .
$$

Proof. Fix $\alpha$ as in the statement. For any $m \geq m_{0}-1$ let

$$
\mathcal{C}_{m}=\left\{\beta \subset\left[m_{0}, \infty\right)|| \beta|=\infty, \alpha \cap[0, m]=\beta \cap[0, m],| \alpha \backslash \beta \mid=\infty\right\} .
$$

Clearly this set is non empty. For each $m \geq m_{0}-1$, pick $\beta(m) \in \mathcal{C}_{m}$. Let $T(m): E_{\beta(m)} \rightarrow E_{\alpha}$ denote the natural (identity) map. By Lemma 8.4 we have $\|T(m)\|_{c b} \geq \sqrt{n} /(2+\varepsilon)^{2}$. However, by Lemma 8.5, for any $k \leq N(m)$ we have $\left\|T(m)_{k}\right\| \leq(2+\varepsilon)$. Assume now that $E_{\alpha}$ is $C$-exact, so that for some finite $k$ there is $F \subset M_{k}$ with $d_{c b}\left(E_{\alpha}, F\right) \leq C$. Choosing $m$ large enough we may ensure that $k \leq N(m)$. By the Smith Lemma 1.1, this implies that $\|T(m)\|_{c b} \leq C\left\|T(m)_{k}\right\|$. Thus we obtain $\sqrt{n} /(2+\varepsilon)^{2} \leq C(2+\varepsilon)$, and hence $C \geq \sqrt{n} /(2+\varepsilon)^{3}$.

Recapitulating, we can now conclude 
Theorem 8.7. For any $n \geq 1$ and $\varepsilon>0$, there is a continuum of $n$-dimensional, subexponential (with constant $2+\varepsilon$ ) operator spaces with mutual cb-distance $\geq n /(2+\varepsilon)^{4}$ and with exactness constant $\geq \sqrt{n} /(2+\varepsilon)^{3}$.

Note that the preceding lower bounds are not significant for small values of $n$.

Remark 8.8. Note that the preceding result can also be obtained using the main idea of [22]. In fact that idea proves more generally that for $\alpha$ infinite with infinitely many gaps the space $E_{\alpha}$ has a large $d_{f}$ constant of embedding into $C^{*}\left(\mathbb{F}_{2}\right)$ in the sense of [21, p. 345].

Corollary 8.9. For any $\varepsilon>0$ there is a separable (infinite dimensional) non-exact operator space which is $(2+\varepsilon)$-subexponential, more precisely such that any finite dimensional subspace $E \subset X$ satifies $K_{E}(N, 2+\varepsilon) \in O\left(N^{2}\right)$.

Proof. Let $E(n)$ be any one of the $n$-dimensional spaces appearing in Theorem 8.7 , Let $X$ be the direct sum in the $c_{0}$-sense of $\{E(n)\}$, so that the elements of $X$ are sequences $x=x(n)$ tending to zero in norm and $X \subset \oplus_{n} E(n)$. We claim that any finite dimensional subspace $E \subset X$ is subexponential with constant $2+\varepsilon$. By perturbation, it suffices to show this for $E=\oplus_{[0 \leq n \leq q]} E(n)$, for any integer $q$. But now an easy verification shows that for such an $E$ we have

$$
K_{E}(N, C) \leq \sum_{0 \leq n \leq q} K_{E(n)}(N, C)
$$

and since each $E(n)$ is subexponential with constant $2+\varepsilon$, we conclude that $E$ also is.

We will now replace our Gaussian random matrices by random unitary ones. Although the picture is less precise, we obtain some information using a rather soft comparison principle. Let $U_{j}^{(m)}$ be an i.i.d. sequence of random unitary matrices uniformly distributed over the unitary group $U(m)$. There is a known domination of $\left\{U_{j}^{(m)}\right\}$ by $\left\{Y_{j}^{(m)}\right\}$ (up to a universal constant) that will allow us to obtain results similar to what precedes for $\left\{U_{j}^{(m)}\right\}$ in place of $\left\{Y_{j}^{(m)}\right\}$.

Fix an integer $n$. Let $D_{Y}(m)(\omega)$ (resp. $\left.D_{U}(m)(\omega)\right)$ be defined by

$$
D_{Y}(m)(\omega)=\sup _{k \leq\left(m / c_{\varepsilon} n\right)^{1 / 2}}\left\{\left\|\sum a_{j} \otimes Y_{j}^{(m)}\right\| \mid a=\left(a_{j}\right) \in M_{k}^{n}\|a\|_{R C} \leq 1\right\}
$$

and

$$
D_{U}(m)(\omega)=\sup _{k \leq\left(m / c_{\varepsilon} n\right)^{1 / 2}}\left\{\left\|\sum a_{j} \otimes U_{j}^{(m)}\right\| \mid a=\left(a_{j}\right) \in M_{k}^{n}\|a\|_{R C} \leq 1\right\},
$$

where $c_{\varepsilon}$ is as in Lemma 8.1. As we saw in Lemma 8.1, we have for a.a. $\omega$ (in fact for all $\omega \in \Omega_{2}$ ) $\lim \sup _{m \rightarrow \infty} D_{Y}(m)(\omega) \leq 2+\varepsilon$ and a fortiori $\sup _{m} D_{Y}(m)(\omega)<\infty$. A close look at the proof of Lemma 0.1 (see also (4.5) ) shows that we have actually for any $p \geq 1$

$$
\mathbb{E} \sup _{m} D_{Y}(m)^{p}<\infty
$$

and hence by dominated convergence

$$
\lim _{m^{\prime} \rightarrow \infty}\left(\mathbb{E} \sup _{m \geq m^{\prime}} D_{Y}(m)^{p}\right)^{1 / p} \leq 2+\varepsilon .
$$

The domination principle of $\left\{U_{j}^{(m)}\right\}$ by $\left\{Y_{j}^{(m)}\right\}$ described in [15, p. 84] implies that for any $p \geq 1$ we have for all integers $q$

$$
\left(\mathbb{E} \sup _{m \geq q} D_{U}(m)^{p}\right)^{1 / p} \leq \chi\left(\mathbb{E} \sup _{m \geq q} D_{Y}(m)^{p}\right)^{1 / p}
$$


where $\chi$ is a numerical constant. Thus we obtain for any $p \geq 1$

$$
\lim _{q \rightarrow \infty}\left(\mathbb{E} \sup _{m \geq q} D_{U}(m)^{p}\right)^{1 / p} \leq \chi(2+\varepsilon),
$$

and a fortiori for a.a. $\omega$

$$
\limsup _{m \rightarrow \infty} D_{U}(m)(\omega) \leq \chi(2+\varepsilon) .
$$

Using this it is immediate that Lemma 8.3 remains valid for $\left\{U_{j}^{(m)}\right\}$ in place of $\left\{Y_{j}^{(m)}\right\}$ provided we replace $2+\varepsilon$ by $\chi(2+\varepsilon)$. Note that in the unitary setting the free circular family $\left(c_{j}\right)$ should be replaced by a free (actually $*$-free) sequence of Haar unitaries.

Let us denote by $E_{U}\left(\alpha_{2}\right)(\omega)$ the space appearing in Lemma 8.3 with $\left\{U_{j}^{(m)}\right\}$ in place of $\left\{Y_{j}^{(m)}\right\}$. Let $K_{N}^{\prime}$ be the largest integer such that $N^{-1 / 2} K_{N}^{\prime}<\left(c_{\varepsilon} n\right)^{-1 / 2}$. Let $\lambda_{N}(\omega)$ be the smallest number $\lambda$ such that $N^{-1 / 2} K_{E_{U}\left(\alpha_{2}\right)(\omega)}(N, \lambda)<\left(c_{\varepsilon} n\right)^{-1 / 2}$ or equivalently such that $K_{E_{U}\left(\alpha_{2}\right)(\omega)}(N, \lambda) \leq K_{N}^{\prime}$. In other words,

$$
\lambda_{N}(\omega)=\inf \left\{d_{N}\left(E_{U}\left(\alpha_{2}\right)(\omega), \hat{E}\right) \mid \hat{E} \subset M_{K_{N}^{\prime}}\right\} .
$$

Note that using the separability of the various underlying metric spaces involved, one can rewrite the preceding infimum (as well as $d_{N}$ ) as a countable infimum, yielding the measurability of $\lambda_{N}$. We then let

$$
\lambda(\omega)=\liminf _{N \rightarrow \infty} \lambda_{N}(\omega) .
$$

Lemma 8.10. With the preceding notation, for a.a. $\omega$

$$
\sqrt{n} \leq \chi(2+\varepsilon) \lambda(\omega),
$$

and hence whenever $C<\sqrt{n}(\chi(2+\varepsilon))^{-1}$ we have

$$
\liminf _{N \rightarrow \infty} N^{-1 / 2} K_{E_{U}\left(\alpha_{2}\right)(\omega)}(N, C) \geq\left(c_{\varepsilon} n\right)^{-1 / 2} .
$$

Proof. Recall that if we set

$$
\Omega_{2}^{U}=\left\{\omega \in \Omega \mid \limsup _{m \rightarrow \infty} D_{U}(m)(\omega) \leq \chi(2+\varepsilon)\right\},
$$

we have $\mathbb{P}\left(\Omega_{2}^{U}\right)=1$. Fix $\omega \in \Omega_{2}^{U}$. Let $N(m)$ be as defined in (8.3). Let $v_{j}(m)=U_{j}^{(N(m))}$ and $v_{j}=\oplus_{m \in \alpha_{2}} v_{j}(m)$, so that $E_{U}\left(\alpha_{2}\right)=\operatorname{span}\left\{v_{1}, \ldots, v_{n}\right\}$. For simplicity let $E=E(\omega)=E_{U}\left(\alpha_{2}\right)$. Fix $m$. Let $K=K_{E_{U}\left(\alpha_{2}\right)(\omega)}\left(m, \lambda_{m}\right)$. By definition of $\lambda_{m}$ we have $c_{\varepsilon} n K^{2}<m$. Moreover, there is $\hat{E} \subset M_{K}$ such that $d_{m}(E, \hat{E}) \leq \lambda_{m}(\omega)$. So we can find a linear isomorphism $w: E \rightarrow \hat{E}$ such that $\left\|w_{m}\right\| \leq 1$ and $\left\|\left(w^{-1}\right)_{m}\right\| \leq \lambda_{m}$. Let $v_{j}^{\prime}=w\left(v_{j}\right)$. Note that $\left\|v_{j}^{\prime}\right\| \leq 1$ for any $j$ (since $v_{j}$ is unitary). We have

$$
n=\left\|\sum v_{j}(m) \otimes \overline{v_{j}(m)}\right\| \leq\left\|\sum v_{j} \otimes \overline{v_{j}(m)}\right\| \leq \lambda_{m}(\omega)\left\|\sum v_{j}^{\prime} \otimes \overline{v_{j}(m)}\right\|,
$$

and hence since $K \leq\left[\sqrt{m / c_{\varepsilon} n}\right]$ and $v_{j}^{\prime} \in M_{K}$ we have

$$
n \leq\left\|\sum v_{j}^{\prime} \otimes \overline{v_{j}(m)}\right\| \leq \lambda_{m} D_{U}\left(\left[\sqrt{m / c_{\varepsilon} n}\right]\right)\left\|\left(v_{j}^{\prime}\right)\right\|_{R C} \leq \lambda_{m} D_{U}\left(\left[\sqrt{m / c_{\varepsilon} n}\right]\right) \sqrt{n},
$$

therefore since $\omega \in \Omega_{2}$

$$
n \leq \liminf _{m} \lambda_{m} \limsup _{m} D_{U}\left(\left[\sqrt{m / c_{\varepsilon} n}\right]\right) \sqrt{n} \leq \lambda \chi(2+\varepsilon) \sqrt{n},
$$

and hence $\sqrt{n} \leq \lambda \chi(2+\varepsilon)$. 
Thus as a recapitulation we may state:

Theorem 8.11. The space $E(\omega)=E_{U}\left(\alpha_{2}\right)(\omega)$ is a.s. $\chi(2+\varepsilon)$-subexponential but not $C$-exact, whenever $C<\sqrt{n}(\chi(2+\varepsilon))^{-1}$. Moreover if $n$ is chosen sufficiently large (so that, say $\sqrt{n}(\chi(2+$ $\varepsilon))^{-1}>3$ ) and $\varepsilon<1$, then a.s.

$$
\underset{N}{\limsup } K_{E(\omega)}(N, 3) / N^{2}<\infty \quad \text { and } \quad \liminf _{N} K_{E(\omega)}(N, 3) / N^{1 / 2}>0
$$

Remark 8.12. A similar result can be proved with large probability for the space $E_{Y}\left(\alpha_{2}\right)(\omega)$ but the fact that $\left\|v_{j}\right\|=1$ makes the preceding result easier to check, so we do not give more details.

\section{Beyond exact or subexponential}

The preceding results highlight the fact that

$$
\left\|\left(a_{1}, \cdots, a_{n}\right)\right\|\left\|=\lim \sup _{N \rightarrow \infty}\right\| \sum_{1}^{n} Y_{j}^{(N)} \otimes a_{j} \|,
$$

is equal to $\left\|\sum c_{j} \otimes a_{j}\right\|$ when $E=\operatorname{span}\left[a_{j}\right]$ is exact or subexponential with constant 1 . It is natural to wonder what happens when $E$ is arbitrary. In this section we make a preliminary study in this direction. In particular, we will see that it is rather easy to estimate (9.1) when $E=\ell_{1}^{n}$ (with maximal o.s.s.) or when $E=O H_{n}$. We also propose a general rough estimate based on the numbers $K_{E}(N, C)$.

Theorem 9.1. Let $\left[a_{i j}\right]$ be any $n \times n$-matrix with complex entries.

(i) Let $U_{i}$ denote the free unitary generators in $C^{*}\left(\mathbb{F}_{n}\right)$. Let $a_{j}=\sum_{i} a_{i j} U_{i}$. Then

$$
2^{-1} \sum_{i}\left(\sum_{j}\left|a_{i j}\right|^{2}\right)^{1 / 2} \leq\left\|||\left(a_{1}, \cdots, a_{n}\right)\right\| \mid \leq 2 \sum_{i}\left(\sum_{j}\left|a_{i j}\right|^{2}\right)^{1 / 2}
$$

(ii) Let $T_{i}$ be any orthonormal basis in $O H_{n}$. Let $a_{j}=\sum_{i} a_{i j} T_{i}$. Then

$$
\left(\sum_{i j}\left|a_{i j}\right|^{2}\right)^{1 / 2} \leq\left\|||\left(a_{1}, \cdots, a_{n}\right)\right\| \mid \leq 2\left(\sum_{i j}\left|a_{i j}\right|^{2}\right)^{1 / 2}
$$

Proof. (i) By the triangle inequality we have $\left\|\left(a_{1}, \cdots, a_{n}\right)\right\| \leq \sum_{i} \lim \sup _{N \rightarrow \infty}\left\|\sum_{j} a_{i j} Y_{j}^{(N)}\right\|$. Let $W_{i}^{(N)}=\sum_{j} a_{i j} Y_{j}^{(N)}$. Since $W_{i}^{(N)}$ has the same distribution as $\left(\sum_{j}\left|a_{i j}\right|^{2}\right)^{1 / 2} Y^{(N)}$ and since $\lim \sup _{N \rightarrow \infty}\left\|Y^{(N)}\right\|=2$, we obtain the upperbound in (i). Let $\left[b_{i j}\right]$ be such that $\sup _{i}\left(\sum_{j}\left|b_{i j}\right|^{2}\right)^{1 / 2} \leq$ 1. To prove the lower bound, it suffices to show that $2^{-1}\left|\sum_{i j} \overline{b_{i j}} a_{i j}\right| \leq\left\|\left|\left(a_{1}, \cdots, a_{n}\right) \|\right|\right.$. Let $Z_{i}^{(N)}=\sum_{k} b_{i k} Y_{k}^{(N)}$. Note that since $Z_{i}^{(N)}$ has the same distribution as $\left(\sum_{j}\left|b_{i j}\right|^{2}\right)^{1 / 2} Y^{(N)}$ we have $\lim \sup _{N \rightarrow \infty}\left\|Z_{i}^{(N)}\right\| \leq 2$. We have clearly

$$
\left|\sum_{i j} a_{i j} \tau_{N}\left(Y_{j}^{(N)} Z_{i}^{(N)^{*}}\right)\right| \leq\left\|\sum_{i j} a_{i j} Y_{j}^{(N)} \otimes \overline{Z_{i}^{(N)}}\right\| \leq \sup _{i}\left\|Z_{i}^{(N)}\right\|\left\|\sum_{i j} a_{i j} Y_{j}^{(N)} \otimes U_{i}\right\|
$$

and hence taking the limsup of both sides when $N \rightarrow \infty$ we find by (0.7)

$$
\left|\sum_{i j} \overline{b_{i j}} a_{i j}\right| \leq 2||\left|\left(a_{1}, \cdots, a_{n}\right)\right|||
$$


which proves (i).

(ii) Let $W_{i}^{(N)}$ be as above. Note that $\left\|\sum_{i j} a_{i j} Y_{j}^{(N)} \otimes T_{i}\right\|=\left\|\sum W_{i}^{(N)} \otimes \overline{W_{i}^{(N)}}\right\|^{1 / 2}$. Therefore $\left|\sum_{i} \tau_{N}\left(W_{i}^{(N)} W_{i}^{(N)^{*}}\right)\right|^{1 / 2} \leq\left\|\sum_{j} Y_{j}^{(N)} \otimes a_{j}\right\|$ and hence taking the limsup of both sides when $N \rightarrow \infty$ we find the lower bound in (ii). For the converse, note that $\left\|\sum W_{i}^{(N)} \otimes \overline{W_{i}^{(N)}}\right\|^{1 / 2} \leq\left(\sum\left\|W_{i}^{(N)}\right\|^{2}\right)^{1 / 2}$ and hence taking the limsup of both sides when $N \rightarrow \infty$ we find the upperbound in (ii).

Recall first that by (1.4) $\left\|\sum_{j} \Delta_{j} \otimes a_{j}\right\|$ is equivalent, up to a factor 2 , to $\left\|\sum_{j} c_{j} \otimes a_{j}\right\|$. Thus, the preceding should be compared with the following:

$$
\left\|\sum_{j} \Delta_{j} \otimes \sum_{i} a_{i j} U_{i}\right\|=\pi_{2}\left(\left[a_{i j}\right]: \ell_{\infty}^{n} \rightarrow \ell_{2}^{n}\right) \quad \text { and } \quad\left\|\sum_{j} \Delta_{j} \otimes \sum_{i} a_{i j} T_{i}\right\|=\left\|\left[a_{i j}\right]\right\|_{4}
$$

where $\pi_{2}$ denotes the 2 -summing norm and $\|\cdot\|_{4}$ the norm in the Schatten class of index 4 . Note that, by the little Grothendieck theorem (see e.g. [23]), there is an absolute constant, namely $\gamma(1)^{-1}$, such that $\left\|\left[a_{i j}\right]: \ell_{\infty}^{n} \rightarrow \ell_{2}^{n}\right\| \leq \pi_{2}\left(\left[a_{i j}\right]: \ell_{\infty}^{n} \rightarrow \ell_{2}^{n}\right) \leq \gamma(1)^{-1}\left\|\left[a_{i j}\right]: \ell_{\infty}^{n} \rightarrow \ell_{2}^{n}\right\|$. We refer the reader to e.g. [23, $\S 5$ and Th. 13.10] for this fact and for the first formula in (9.2) We refer to [20, p. 38] for the second one.

Remark 9.2. We end this paper with a majorization that can be applied to estimate ||$\left|\left(a_{1}, \cdots, a_{n}\right)\right|||$ for arbitrary $a_{j} \in B(H)$. This illustrates the possible applications of bounds such as (4.5). Let $E$ denote the linear span of an arbitrary $n$-tuple $\left(a_{1}, \cdots, a_{n}\right)$ in $B(H)$. Let $i_{E}: E \rightarrow B(H)$ denote the inclusion of $E$ into $B(H)$. Fix $N \geq 1$. Assume that we have sequences of maps $v(m): E \rightarrow M_{K(m)}$, $w(m): M_{K(m)} \rightarrow B(H)$ such that $\sum_{m}\|w(m)\|\|v(m)\|<\infty$ and $i_{E}=\sum_{m} w(m) v(m)$. Then the following is an immediate consequence of (4.5).

$$
\mathbb{E}\left\|\sum_{1}^{n} Y_{j}^{(N)} \otimes a_{j}\right\| \leq(1+\varepsilon) \sum_{m}\left(2+\gamma_{\varepsilon}\left(\frac{\log (K(m))+1}{N}\right)^{1 / 2}\right) \|\left(w(m)_{N}\left\|\left(v(m)\left(a_{j}\right)\right)\right\|_{R C} .\right.
$$

Acknowledgment. I thank Kate Juschenko for useful conversations at an early stage of this investigation. I am very grateful to Mikael de la Salle for numerous remarks and suggestions that led to many improvements. Thanks are due also to Yanqi Qiu, Alexandre Nou and the anonymous referee for several corrections.

\section{References}

[1] N.P. Brown and N. Ozawa, $C^{*}$-algebras and finite-dimensional approximations, Graduate Studies in Mathematics, 88, American Mathematical Society, Providence, RI, 2008.

[2] A. Buchholz, Operator Khintchine inequality in non-commutative probability. Math. Ann. 319 (2001), 1-16.

[3] B. Collins and C. Male, The strong asymptotic freeness of Haar and deterministic matrices, To appear.

[4] E.G. Effros and Z.J. Ruan, Operator Spaces, The Clarendon Press, Oxford University Press, New York, 2000, xvi+363 pp.

[5] T. Figiel, J. Lindenstrauss and V. Milman. The dimension of almost spherical sections of convex bodies. Acta Math. 139 (1977), 53-94. 
[6] S. Geman, A Limit Theorem for the Norm of Random Matrices, Ann. Prob., 8 (1980), 252261.

[7] M. Gromov and V. Milman, A Topological Application of the Isoperimetric Inequality, American Journal of Mathematics, 105 (1983), 843-854.

[8] U. Haagerup and S. Thorbjørnsen, Random matrices and $K$-theory for exact $C^{*}$-algebras, Doc. Math. 4 (1999), 341-450 (electronic).

[9] U. Haagerup and S. Thorbjørnsen, A new application of random matrices: $\operatorname{Ext}\left(C_{\text {red }}^{*}\left(\mathbb{F}_{2}\right)\right)$ is not a group. Ann. of Math. 162 (2005), 711-775.

[10] U. Haagerup, H. Schultz and S. Thorbjørnsen, A random matrix approach to the lack of projections in $C_{\text {red }}^{*}\left(\mathbb{F}_{2}\right)$. Adv. Math. 204 (2006), no. 1, 1-83.

[11] U. Haagerup and M. Musat, The Effros-Ruan conjecture for bilinear forms on $C^{*}$-algebras, Invent. Math. 174 (2008), 139-163.

[12] M. Junge and G. Pisier, Bilinear forms on exact operator spaces and $B(H) \otimes B(H)$, Geom. Funct. Anal. 5 (1995), no. 2, 329-363.

[13] M. Ledoux, The Concentration of Measure Phenomenon, American Mathematical Society, Providence, RI, 2001.

[14] C. Male, The norm of polynomials in large random and deterministic matrices with an appendix by Dimitri Shlyakhtenko, arXiv:1004.4155v5

[15] M.B. Marcus and G.Pisier, Random Fourier series with Applications to Harmonic Analysis. Annals of Math. Studies $n^{\circ} 101$, Princeton University Press. (1981).

[16] T. Oikhberg and É. Ricard, Operator spaces with few completely bounded maps. Math. Ann., 328 (2004) 229-259.

[17] G. Pisier, Remarques sur un résultat non publié de B. Maurey. Seminar on Functional Analysis, 1980-1981, Exp. No. V, 13 pp., École Polytech., Palaiseau, 1981. [Available at http://www.numdam.org/numdam-bin/feuilleter?j=SAF

[18] G. Pisier, Probabilistic methods in the geometry of Banach spaces, Probability and Analysis (Varenna, 1985), 167-241, Lecture Notes in Math., 1206, Springer, Berlin, 1986.

[19] G. Pisier, The volume of Convex Bodies and Banach Space Geometry. Cambridge University Press, 1989.

[20] G. Pisier, The operator Hilbert space OH, complex interpolation and tensor norms, Mem. Amer. Math. Soc. 122 (1996), no. 585.

[21] G. Pisier, Introduction to operator space theory, Cambridge University Press, Cambridge, 2003.

[22] G. Pisier, Remarks on $B(H) \otimes B(H)$. Proc. Indian Acad. Sci. 116 (2006), 423-428.

[23] G. Pisier, Grothendieck's theorem, past and present. Bull. Amer. Math. Soc. 49 (2012), 237323. 
[24] G. Pisier, Martingale inequalities and Operator space structures on $L_{p}$, Preprint, 2012.

[25] G. Pisier, Quantum expanders and geometry of operator spaces, To appear.

[26] G. Pisier and D. Shlyakhtenko, Grothendieck's theorem for operator spaces, Invent. Math. 150 (2002), no. 1, 185-217.

[27] O. Regev and T. Vidick, A simple proof of Grothendieck's theorem for completely bounded norms, Preprint 2012, to appear in J. Op. Theory.

[28] H. Schultz, Non-commutative polynomials of independent Gaussian random matrices, The real and symplectic cases, Probab. Theory Rel. Fields 131 (2005) 261309.

[29] D. Voiculescu, K. Dykema and A. Nica, Free random variables, American Mathematical Society, Providence, RI, 1992. 